\title{
Minimum cost connection networks: truth-telling and implementation
}

\author{
Jens Leth Hougaard* $\quad$ Mich Tvede ${ }^{\dagger}$
}

\begin{abstract}
In the present paper we consider the allocation of costs in connection networks. Agents have connection demands in form of pairs of locations they want to have connected. Connections between locations are costly to build. The problem is to allocate costs of networks satisfying all connection demands. We use a few axioms to characterize allocation rules that truthfully implement cost minimizing networks satisfying all connection demands in a game where: (1) a central planner announces an allocation rule and a cost estimation rule; (2) every agent reports her own connection demand as well as all connection costs; (3) the central planner selects a cost minimizing network satisfying reported connection demands based on the estimated costs; and, (4) the planner allocates the true costs of the selected network. It turns out that an allocation rule satisfies the axioms if and only if relative cost shares are fixed.
\end{abstract}

Keywords: Axiomatic characterization; Connection networks; Cost sharing, Implementation; Truth-telling.

JEL Classification: C70, C72, D71, D85.

*IFRO, University of Copenhagen, Rolighedsvej 25, DK-1958 Frederiksberg C, Denmark, phone: +45 35 3368 14, email: jlh@ifro.ku.dk

${ }^{\dagger}$ Newcastle University, 5 Barrack Road, Newcastle upon Tyne, NE1 4SE, United Kingdom, phone: +44 (0)191 208 1643, email: mich.tvede@ncl.ac.uk 


\section{Introduction}

Overview of the paper: In the present paper we consider cost allocation in the connection network (CN) model used in Anshelevich et al. (2008), Chen et al. (2010), Juarez \& Kumar (2013) and Moulin (2014). There are finite sets of locations and agents. Agents have connection demands in form of pairs of locations they want to have directly or indirectly connected. Connections between locations are costly, undirected and free from congestion. Consequently, several agents can use the same connection as part of paths satisfying their connection demands. Therefore connections are public goods. The $\mathrm{CN}$ model has broad empirical relevance as illustrated in Section 6 where we discuss the German Hansa as an example of network building and cost allocation in practice.

A minimum cost connection network ( $\mathrm{MCCN}$ ) is a network minimizing total cost subject to the constraint that all connection demands have to be satisfied. An allocation rule maps MCCNs, connection demands and connection costs to cost shares for all agents. Depending on the allocation rule there can be a potential conflict between overall welfare aimed at minimizing total cost and individual welfare aimed at minimizing individual cost shares. We characterize the set of allocation rules that truthfully implement MCCNs.

Two properties are at heart of our characterization: Unobserved Information Independence (UII); and, Network Independence (NI). UII states that connection costs of unused connections should not influence cost shares. Two versions of UII are considered: UII for comparison of situations with the same set of locations and Strong UII (SUII) for comparison of situations with different sets of locations. NI states that cost shares should not depend on the selected MCCN in case of multiple MCCNs. In addition, Scale Invariance (SI) stating that cost shares are homogeneous of degree one in connection costs is considered. We characterize allocation rules satisfying UII or SUII and NI (Theorem 1 and Corollary 1) as well as UII or SUII, NI and SI (Corollaries 2 and 3).

The difference between UII and SUII is small. However, whether we use UII or SUII has some impact on the domain as well as the form of allocation rules. With UII we have to focus on connection networks with undemanded locations and allocation rules can depend on the sets of locations. With SUII we can consider all CNs and allocation rules cannot depend on the sets of locations.

In Theorem 1 and Corollary 1 we show that an allocation rule satisfies UII or SUII and NI if and only if cost shares depend on connection demands, total cost, the set of locations in case of UII, and nothing else. In Corollaries 2 and 3 we show that an allocation rule satisfies UII or SUII, NI and SI if and only if relative cost shares depend on connection demands, the set of locations in case of UII, and nothing else. Theorem 1 implies UII and NI are at odds with Individual Rationality where Individual Rationality means that no agent pays 
more than the cost of building a path satisfying her own connection demand.

In order to consider truthful implementation of MCCNs in the $\mathrm{CN}$ model we consider a variation of the game introduced in Hougaard \& Tvede (2012) where a planner is ignorant of connection demands and connection costs:

(1) A planner announces a cost allocation rule and a cost estimation rule.

(2) Every agent reports her connection demand and all connection costs.

(3) The planner selects a MCCN based on reported connection demands and estimated connection costs.

(4) The planner allocates the true costs of the selected network.

Agents connection cost reports are used by the planner to estimate connection costs. The planner selects a MCCN based on estimated connection costs. True connection costs for connections in the selected MCCN are observed. The planner allocates the true costs of the selected network. Agents can misreport connection costs. However misreporting is not revealed for connections not in the selected MCCN.

We show that if an allocation rule truthfully implement MCCNs, then it satisfies UII and NI (Observations 1 and 2); and, we characterize allocation rules satisfying UII and NI as well as UII, NI and SI that truthfully implements MCCNs (Theorem 2 and Corollary 4). Consequently, voluntary participation - even in the weak form of Individual Rationality - is at odds with truthful implemention of MCCNs. Therefore economic efficiency can be attained if and only if the planner is able to prevent agents from building their own networks. Moreover, in Theorem 3 we address the issue of how the planner being less ignorant, knowing either connection demands or connection costs, influences the set of networks that can be implemented. Finally, we extend the characterization of allocation rules satisfying UII, NI and SI and truthfully implementing MCCNs to the case of incomplete information (Corollary 5).

In the minimum cost spanning tree (MCST) model one location is a source and every other location is inhabited by an agent. Every agent wants to be connected to the source making the MCST model a special case of the CN model. For the MCST model it has been shown (Hougaard and Tvede, 2012) that the set of allocation rules satisfying reductionism (a strong form of UII) and monotonicity (a strong form of NI) contains fixed relative cost share rules such as the equal split rule, the folk rule discussed in Bogomolnaia \& Moulin (2010) and the rest of the family of obligation rules introduced and analyzed in Tijs et al. (2006). The family of obligation rules are Stand Alone core stable where Stand alone core stable means that no coalition of agents pays more than the cost of building a network satisfying the connection demands of its members. Hence UII and NI are compatible with Stand Alone core stability in the MCST model. In contrast, UII and NI are incompatible with Individual 
Rationality in the $\mathrm{CN}$ model as mentioned above.

Related literature: Implementation in the $\mathrm{CN}$ model has been analyzed in Anshelevich et al. (2008), Chen et al. (2010) and Juarez \& Kumar (2013). The same game is used in all three papers:

(1) A central planner announces a cost allocation rule.

(2) Every agent reports a path between the pair of locations she wants to be connected.

(3) The central planner selects the network consisting of all reported paths and allocates costs of the selected network.

In comparison with our game, every agent has less impact on the network selection, because the paths reported by the other agents are parts of the selected network. The planner has more information than in our game, because the planner knows connection demands and connection costs. In Anshelevich et al. (2008) and Chen et al. (2010) properties of the connectionwise equal split rule (the cost of every connection is divided equally between agents whose reported paths include the connection) are studied. It is shown that: Nash equilibria exist; all Nash equilibria can be inefficient; and, there are bounds on the ratios between the cost of the cheapest Nash equilibrium and the cost of a MCCN (price of stability) and the cost of the most expensive Nash equilibrium and the cost of a MCCN (price of anarchy). In Juarez \& Kumar (2013) attention is restricted to the set of allocation rules that depend on costs of reported paths, total cost and nothing else. It is shown that the set of allocation rules implementing MCCNs and the set of fixed relative cost shares rules are identical. In comparison with the present paper a much smaller set of allocation rules is considered. Since attention is restricted to allocation rules that do not depend on connection demands, our Corollary 2 implies that the only allocation rules satisfying UII, NI and SI are fixed relative cost shares rules.

Implementation in the MCST model has been analyzed in Bergantinos \& Lorenzo (2004, 2005), Bergantinos \& Vidal-Puga (2010) and Hougaard \& Tvede (2012). In all four papers existence of Nash equilibria and their properties are considered. In comparison with Hougaard and Tvede (2012), the present paper illustrates the drastic consequences of going from the MCST model to the more general CN model. It was shown in Megiddo (1978) and Tamir (1991) that the set of Stand Alone core stable allocations can be empty for the MCST model (and therefore also the $\mathrm{CN}$ model) with undemanded locations. Hence UII and NI are trivially incompatible with Stand Alone core stability. However, the implications of Theorems 1 and 2 are less trivial: in the CN model UII and NI as well as truthful implementation are incompatible with Individual Rationality. In Moulin (2014) the folk rule is extended from the MCST model to two subclasses of the $\mathrm{CN}$ model for which the set of Stand Alone core stable allocations is nonempty. 
Plan of the paper: In Section 2 we introduce the set up and our axioms; in Section 3 we characterize allocation rules satisfying our axioms; in Section 4 we study implementation of MCCNs; in Section 5 we study implementation of MCCNs in case of incomplete information; in Section 6 we discuss the German Hansa; and finally, in Section 7 we end with a few remarks.

\section{The CN model}

In the present section we introduce our set up and axioms.

\section{Set up}

Let $\mathscr{M}=\{1, \ldots, m\}$ be a set of finitely many agents and $\mathscr{L}$ a set of possible locations with $|\mathscr{L}| \geq 2 m+1$. The set of possible locations $\mathscr{L}$ can be finite or infinite. We focus on the allocation of costs in connection networks with finitely many locations $\mathscr{N}$ selected from the set of possible locations $\mathscr{L}$. The set of connections between pairs of possible locations is $\mathscr{L}^{2}=\mathscr{L} \times \mathscr{L}$.

Every agent $i \in \mathscr{M}$ has a connection demand in form of a pair of locations $\left(a_{i}, b_{i}\right) \in \mathscr{L}^{2}$ she wants to be directly or indirectly connected. A connection structure $P$ is a collection of individual connection demands $\left(a_{i}, b_{i}\right)_{i \in \mathscr{M}}$. Let $\mathscr{P}$ be the set of connection structures. A cost structure $C$ describes costs of connecting locations and is defined by a set of finitely many locations $\mathscr{N}_{C} \subset \mathscr{L}$ and a map $c: \mathscr{N}_{C}^{2} \rightarrow \mathbb{R}_{+}$with

- $c_{j j}=0$ for all $j$.

- $c_{j k}>0$ for all $j$ and $k$ with $j \neq k$.

- $c_{k j}=c_{j k}$ for all $j$ and $k$.

Connections are undirected and free from congestion. For $2^{\mathscr{L}}$ being the set of subsets of $\mathscr{L}$ let $\mathscr{F} \subset \mathscr{P} \times 2^{\mathscr{L}}$ be the set of connection structures $P$ and subsets of locations $\mathscr{N}$ with $\left(a_{i}, b_{i}\right) \in \mathscr{N}^{2}$ for all $i$. A connection problem is a connection structure and a cost structure $(P, C)$ with $\left(P, \mathscr{N}_{C}\right) \in \mathscr{F}$.

A graph $g$ on $\mathscr{N}$ is a set of locations and a set of connections $(V, E)$ with $V \subset \mathscr{N}$ and $E \subset V \times V$. A CN for a connection problem $(P, C)$ is a graph $g=(V, E)$ such that for every agent $i$ there is a path $p_{i}=\left(V_{i}, E_{i}\right) \subset g$ where $V_{i}=\left\{a_{i}, r, r^{\prime}, \ldots, s, s^{\prime}, b_{i}\right\}$ and $E_{i}=$ $\left\{a_{i} r, r r^{\prime}, \ldots, s s^{\prime}, s^{\prime} b_{i}\right\}$. CNs can contain cycles. Let $v(g, c)$ be the total cost of a CN

$$
v(g, c)=\sum_{j k \in g} c_{j k} .
$$


A MCCN for a connection problem $(P, C)$ is a $\mathrm{CN} g$ minimizing total cost subject to the constraint that all connection demands have to be satisfied. Therefore $g$ is a MCCN if and only if $v(g, c) \leq v(h, c)$ for every $\mathrm{CN} h$. The set of MCCNs is non-empty and finite because the set of CNs is non-empty and finite. Let $\mathscr{M} \mathscr{C} \mathscr{C} \mathscr{N}(P, C)$ be the set of MCCNs for a given connection problem $(P, C)$. Clearly every $\mathrm{MCCN}$ is either a tree or a forest (a collection of trees). Indeed if a $\mathrm{CN}$ contains a cycle, then removing any connection in the cycle does not change whether connection demands are satisfied or not. Since every MCCN $g$ is either a tree or a forest, for every agent $i$ there is a unique path $p_{i}(g, P)$ between $a_{i}$ and $b_{i}$.

A cost allocation problem $(g, P, C)$ is a $\mathrm{CN} g$ and a connection problem $(P, C)$ such that $g$ is a MCCN for $(P, C)$. Let $\mathscr{U}$ be the set of cost allocation problems $(g, P, C)$ and $\mathscr{U}_{U} \subset \mathscr{U}$ the set of cost allocation problems with undemanded locations so $(g, P, C) \in \mathscr{U}_{U}$ provided $\mathscr{N}_{C} \backslash \cup_{i \in \mathscr{M}}\left\{a_{i}, b_{i}\right\} \neq \emptyset$.

\section{Allocation of costs}

For a given cost allocation problem $(g, P, C)$ the total cost of the MCCN $g$ has to be shared among agents in $\mathscr{M}$. An allocation rule $\phi: \mathscr{U} \rightarrow \mathbb{R}_{+}^{m}$ maps a cost allocation problem to an $m$-dimensional vector of positive cost shares,

$$
\phi(g, P, C)=\left(\phi_{1}(g, P, C), \ldots, \phi_{m}(g, P, C)\right),
$$

for which budget-balance $\sum_{i \in \mathscr{M}} \phi_{i}(g, P, C)=v(g, c)$ is satisfied.

Two examples of allocation rules are the Shapley value considered in Moulin (2013) and the connectionwise equal split (also denoted the Shapley protocol) considered in Chen et al. (2010). Additional notation is needed to define the Shapley value. Let $\mathscr{S}(i) \subset 2^{\mathscr{M}}$ be the set of coalitions of agents which contain agent $i$ and $V_{S}(C)$, where $V_{\emptyset}(C)=0$, the minimum cost for a graph for which connection demands for all agents in $S \in 2^{\mathscr{M}}$ are satisfied. The Shapley value is defined as follows: for all $i$,

$$
\phi_{i}^{S V}(g, P, C)=\sum_{S \in \mathscr{S}(i)} \frac{(|S|-1) !(m-|S|) !}{m !}\left(V_{S}(C)-V_{S \backslash\{i\}}(C)\right) .
$$

The connectionwise equal split is defined as follows: for all $i$,

$$
\phi_{i}^{C E S}(g, P, C)=\sum_{j k \in p_{i}(g, P)} \frac{c_{j k}}{\left|\left\{i^{\prime} \in \mathscr{M} \mid j k \in p_{i^{\prime}}(g, P)\right\}\right|} .
$$

\section{Axioms}

Now we present axioms used to characterize cost allocation rules. The first three properties are at the heart of our characterizations. 
Unobserved Information Independence (UII) For all $(g, P, C),(g, P, D) \in \mathscr{U}$ with $\mathscr{N}_{C}=$ $\mathscr{N}_{D}$ and $c_{j k}=d_{j k}$ for all $j k \in g, \phi(g, P, C)=\phi(g, P, D)$.

UII states that for two cost allocation problems with identical MCCNs, connection structures, sets of locations and observed connection costs, the allocations of costs should be identical. Hence UII implies the allocation of costs is independent of costs of unobserved connections but can depend on the set of locations.

Strong Unobserved Information Independence (SUII) For all $(g, P, C),(g, P, D) \in \mathscr{U}$ with $c_{j k}=d_{j k}$ for all $j k \in g, \phi(g, P, C)=\phi(g, P, D)$.

SUII states that for two cost allocation problems with identical MCCNs, connection structures and observed connection costs, the allocations of costs should be identical. Hence SUII implies the allocation of costs is independent of costs of unobserved connections and the set of locations. Clearly SUII implies UII.

Network Independence (NI) For all $(g, P, C),(h, P, C) \in \mathscr{U}, \phi(g, P, C)=\phi(h, P, C)$.

NI states that for a connection problem with multiple MCCNs, the allocations of costs should be identical for all MCCNs. Hence NI implies the allocation of costs is independent of the chosen MCCN.

Scale Invariance (SI) For all $(g, P, C) \in \mathscr{U}$ and $\delta>0, \phi\left(g, P,\left(\mathscr{N}_{C}, \delta c\right)\right)=\delta \phi(g, P, C)$.

SI is a standard property stating that cost shares are homogeneous of degree one in connection costs.

In case of constructing a network, depending on the perception of fairness, costs of unused connections can be allowed to have more or less influence on the allocation of the total cost of the realized network. The position reflected in SUII as well as UII for problems with identical sets of locations is that since the cost of a realized $\mathrm{CN}$ is observed and costs of unused connections are unobserved, the allocation of costs should not depend on the costs of unused connections. Moreover for allocation rules that allow costs of unused connections to influence the allocation of total cost there is a conflict between agents over cost estimates of unused connections. This conflict can be an obstacle for implementation of the efficient network as we demonstrate below in Observation 1. All in all, SUII can be seen to reflect a positive as well as a normative approach to cost sharing. The connectionwise equal split rule satisfies SUII. Allocation rules violating SUII include the Shapley value.

In connection problems with several MCCNs total cost are identical for all MCCNs. Therefore there is no reason for choosing one MCCN over another. The position reflected 
in NI is that the allocation of costs should not depend on the chosen MCCN. Moreover for allocation rules that allow the choice of MCCN to influence the allocation of costs there is a conflict between agents over which MCCN to choose. This conflict can be an obstacle for implementation of the efficient network as we demonstrate below in Observation 2. All in all, NI can be seen to reflect a positive as well as a normative approach to cost sharing. The Shapley value satisfies NI. Allocation rules violating NI include the connectionwise equal split rule.

For the MCST model we showed in Hougaard \& Tvede (2012) that an allocation rule truthfully implements MCCNs if and only if it is reductionist and monotonic. An allocation rule is reductionist provided it depends on irreducible costs, where the irreducible cost of a connection is: the true cost in case the connection is used; and, the lowest cost for which the connection needs not be used in case the connection is unused. An allocation rule is monotonic provided it is monotonic in irreducible costs. For the $\mathrm{CN}$ model irreducible costs cannot be defined. However: an implication of reductionism is that connection costs of unused connections do not influence cost shares as formalized in SUII; and, an implication of monotonicity is that cost shares are independent of the selected MCCN in case of multiple MCCNs as formalized in NI.

\section{Characterization results}

In the present section we characterize allocation rules satisfying UII or SUII and NI as well as allocation rules satisfying UII or SUII, NI and SI.

\section{Simple allocation rules}

First we consider allocation rules for which cost shares depend on the connection structure $P$, the set of locations $\mathscr{N}_{C}$, the total $\operatorname{cost} v(g, c)$ of the MCCN $g$ and nothing else.

Definition 1 A simple allocation rule is an allocation rule $\phi: \mathscr{U} \rightarrow \mathbb{R}_{+}^{m}$ for which there is a map $\gamma: \mathscr{F} \times \mathbb{R}_{++} \rightarrow \mathbb{R}_{+}^{m}$ such that for all cost allocation problems $(g, P, C) \in \mathscr{U}$,

$$
\phi(g, P, C)=\gamma\left(P, \mathscr{N}_{C}, v(g, c)\right) .
$$

We use UII and NI to characterize simple allocation rules on $\mathscr{U}_{U}$.

Theorem 1 An allocation rule $\phi$ on $\mathscr{U}_{U}$ satisfies UII and NI if and only if it is simple.

In Hougaard \& Tvede (2012) we considered the MCST model where $\mathscr{M}=\{1, \ldots, m\}$, $\mathscr{N}=\{0, \ldots, m\}$ and $\left(a_{i}, b_{i}\right)=(0, i)$ for all $i$. We showed that the set of allocation rules 
satisfying UII and NI is quite large and includes the equal split rule, the folk rule and the rest of the family of obligation rules. All allocation rules in the family of obligation rules are Stand Alone core stable. Theorem 1 implies that adding locations not inhabited by any agent to the MCST model shrinks the set of allocation rules dramatically. Indeed the set of allocation rules satisfying SUII and NI contains no Individually Rational allocation rule for the $\mathrm{CN}$ model with undemanded locations.

Before we provide a proof for Theorem 1 we discuss the implications of replacing UII with SUII and adding SI to UII and NI as well as SUII and NI.

\section{Strongly simple allocation rules}

Second we consider allocation rules for which cost shares depend on the connection structure $P$, the total cost $v(g, c)$ of the MCCN $g$ and nothing else.

Definition 2 A strongly simple allocation rule is an allocation rule $\phi: \mathscr{U} \rightarrow \mathbb{R}_{+}^{m}$ for which there is a map $\lambda: \mathscr{P} \times \mathbb{R}_{++} \rightarrow \mathbb{R}_{+}^{m}$ such that for all cost allocation problems $(g, P, C) \in \mathscr{U}$,

$$
\phi(g, P, C)=\lambda(P, v(g, c)) .
$$

We use SUII and NI to characterize strongly simple allocation rules on $\mathscr{U}$.

Corollary 1 An allocation rule $\phi$ satisfies SUII and NI if and only if it is strongly simple.

Proof: According to Theorem 1 there is a map $\gamma: \mathscr{F} \times \mathbb{R}_{++} \rightarrow \mathbb{R}_{+}^{m}$ such that $\phi(g, P, C)=$ $\gamma\left(P, \mathscr{N}_{C}, v(g, c)\right)$ for all $(g, P, C) \in \mathscr{U}_{U}$ because $\phi$ is satisfies UII and NI on $\mathscr{U}_{U}$. For every pair of cost allocation problems $(g, P, C)$ and $(g, P, D)$ in $\mathscr{U}_{U}$ with $c_{j k}=d_{j k}$ for all $j k \in g$, $\gamma\left(P, \mathscr{N}_{C}, v(g, c)\right)=\gamma\left(P, \mathscr{N}_{D}, v(g, d)\right)$ according to SUII. Hence for every connection structure $P$ and total cost $v$ pick an arbitrary cost allocation problem $(g, P, C) \in \mathscr{U}_{U}$ with $v(g, c)=v$ and let $\lambda(P, v)$ be defined by $\lambda(P, v)=\gamma\left(P, \mathscr{N}_{C}, v(g, c)\right)$. For a cost allocation problem $(g, P, D) \in$ $\mathscr{U} \backslash \mathscr{U}_{U}$ there is a location $r \in \mathscr{L} \backslash \mathscr{N}_{D}$ because $\left|\mathscr{N}_{D}\right| \leq 2 m$ and $|\mathscr{L}| \geq 2 m+1$. Consider a cost allocation problem $(g, P, C)$ with $\mathscr{N}_{C}=\mathscr{N}_{D} \cup\{r\}, c_{j k}=d_{j k}$ for $j k \in g$ and $c_{j k}=v(g, d)$ for $j=r$ and all $k \neq r$. Then $\phi(g, P, D)=\phi(g, P, C)=\lambda(P, v(g, c))=\lambda(P, v(g, d))$. Therefore the allocation rule is strongly simple.

\section{Linear simple allocation rules}

For simple allocation rules, the allocation of costs can depend on total cost in quite weird ways. Indeed, consider the following example: If total cost is a rational number $v(g, c) \in \mathbb{Q}$, 
then the total cost is split equally between agents

$$
\phi_{i}(g, P, C)=\frac{v(g, c)}{m}
$$

for all $i$ and if the total cost is an irrational number $v(g, c) \in \mathbb{R} \backslash \mathbb{Q}$, then the total cost is allocated between agents such that

$$
\phi_{i}(g, P, C)=\frac{w_{i}}{\sum_{j \in \mathscr{M}} w_{j}} v(g, c)
$$

where

$$
w_{i}=\sum_{j \in\left\{a_{i}, b_{i}\right\}}\left(\left|\left\{i^{\prime} \mid j=a_{i^{\prime}}\right\}\right|+\left|\left\{i^{\prime} \mid j=b_{i^{\prime}}\right\}\right|\right)
$$

for all $i$. The rule obviously violates SI although each of the two parts of the rule satisfies SI on its domain. The first part of the cost allocation rule is the equal split rule. The second part is a rule where the cost share of agent $i$ depends on the number of agents who have the locations in the connection demand of agent $i$ as parts of their connection demands.

Third we consider allocation rules for which relative cost shares depend on connection structures $P$, the set of locations $\mathscr{N}_{C}$ and nothing else.

Definition 3 A linear simple allocation rule is an allocation rule $\phi: \mathscr{U} \rightarrow \mathbb{R}_{+}^{m}$ for which there is a map $\Gamma: \mathscr{F} \rightarrow \mathbb{R}_{+}^{m}$ such that for all cost allocation problems $(g, P, C) \in \mathscr{U}$,

$$
\phi(g, P, C)=\Gamma\left(P, \mathscr{N}_{C}\right) v(g, c) .
$$

We use UII, NI and SI to characterize linear simple allocation rules on $\mathscr{U}_{U}$.

Corollary 2 An allocation rule $\phi$ on $\mathscr{U}_{U}$ satisfies UII, NI and SI if and only if it is linear simple.

Proof: We leave it to the reader to check that linear simple allocation rules satisfy UII, NI and SI. Consequently we focus on the converse claim.

According to Theorem 1 if an allocation rule $\phi: \mathscr{U}_{U} \rightarrow \mathbb{R}_{+}^{m}$ satisfies UII and NI, then there is a map $\gamma: \mathscr{F} \times \mathbb{R}_{++} \rightarrow \mathbb{R}_{+}^{m}$ such that for all $(g, P, C) \in \mathscr{U}_{U}, \phi(g, P, C)=\gamma\left(P, \mathscr{N}_{C}, v(g, c)\right)$. For all $(g, P, C) \in \mathscr{U}_{U}$ and $\delta>0, v(g, \delta c)=\delta v(g, c)$. Consider two cost allocations problems $(g, P, C),(h, P, D) \in \mathscr{U}_{U}$ with $\mathscr{N}_{C}=\mathscr{N}_{D}$ and $v(g, c)=\delta v(h, d)$ for some $\delta>0$. Then

$\phi(g, P, C)=\gamma\left(P, \mathscr{N}_{C}, v(g, c)\right)=\gamma\left(P, \mathscr{N}_{C}, \delta v(h, d)\right)=\gamma\left(P, \mathscr{N}_{D}, v(h, \delta d)\right)=\phi\left(h, P,\left(\mathscr{N}_{D}, \delta d\right)\right)$

If the allocation rule satisfies SI, then $\phi\left(h, P,\left(\mathscr{N}_{D}, \delta d\right)\right)=\delta \phi(h, P, D)$. Therefore the relative cost shares are independent of the MCCN and the cost structure

$$
\frac{1}{v(g, c)} \phi(g, P, C)=\frac{1}{\delta v(h, d)} \delta \phi(h, P, D)=\frac{1}{v(h, d)} \phi(h, P, D) .
$$


Hence for every connection structure $P \in \mathscr{P}$ pick an arbitrary cost allocation problem $(h, P, D) \in \mathscr{U}_{U}$ and let $\Gamma\left(P, \mathscr{N}_{D}\right)$ be defined by

$$
\Gamma\left(P, \mathscr{N}_{D}\right)=\frac{1}{v(h, d)} \phi(h, P, D)
$$

Then for all $(g, P, C) \in \mathscr{U}_{U}, \phi(g, P, C)=\Gamma\left(P, \mathscr{N}_{C}\right) v(g, c)$. Thus the allocation rule is linear simple.

\section{Linear strongly simple allocation rules}

Fourth we consider allocation rules for which relative cost shares depend on connection structures $P$ and nothing else.

Definition $4 A$ linear strongly simple allocation rule is an allocation rule $\phi: \mathscr{U} \rightarrow \mathbb{R}_{+}^{m}$ for which there is a map $\Lambda: \mathscr{P} \rightarrow \mathbb{R}_{+}^{m}$ such that for all cost allocation problems $(g, P, C) \in \mathscr{U}$,

$$
\phi(g, P, C)=\Lambda(P) v(g, c) .
$$

We use SUII, NI and SI to characterize linear strongly simple allocation rules on $\mathscr{U}$.

Corollary 3 An allocation rule $\phi$ satisfies SUII, NI and SI if and only if it is linear strongly simple.

Proof: We leave it to the reader to check that linear strongly simple allocation rules satisfy SUII, NI and SI. Consequently we focus on the converse claim.

According to Corollary 1 if an allocation rule $\phi: \mathscr{U} \rightarrow \mathbb{R}_{+}^{m}$ satisfies SUII and NI, then there is a map $\lambda: \mathscr{P} \times \mathbb{R}_{++} \rightarrow \mathbb{R}_{+}^{m}$ such that for all $(g, P, C) \in \mathscr{U}, \phi(g, P, C)=\lambda(P, v(g, c))$. For all $(g, P, C) \in \mathscr{U}$ and $\delta>0, v(g, \delta c)=\delta v(g, c)$. Consider two cost allocations problems $(g, P, C),(h, P, D) \in \mathscr{U}$ with $v(g, c)=\delta v(h, d)$ for some $\delta>0$. Then

$$
\left.\phi(g, P, C)=\lambda(P, v(g, c))=\lambda(P, \delta v(h, d))=\lambda(P, v(h, \delta d))=\phi\left(h, P, \mathscr{N}_{D}, \delta d\right)\right)
$$

If the allocation rule satisfies SI, then $\phi\left(h, P,\left(\mathscr{N}_{D}, \delta d\right)\right)=\delta \phi(h, P, D)$. Therefore the relative cost shares are independent of the MCCN and the cost structure

$$
\frac{1}{v(g, c)} \phi(g, P, C)=\frac{1}{\delta v(h, c)} \delta \phi(h, P, D)=\frac{1}{v(h, d)} \phi(h, P, D) .
$$

Hence for every connection structure $P \in \mathscr{P}$ pick an arbitrary cost allocation problem $(h, P, D) \in \mathscr{U}$ and let $\Lambda(P)$ be defined by

$$
\Lambda(P)=\frac{1}{v(h, d)} \phi(h, P, D)
$$


Then for all $(g, P, C) \in \mathscr{U}, \phi(g, P, C)=\Lambda(P) v(g, c)$. Thus the allocation rule is linear strongly simple.

For linear strongly simple allocation rules, the allocation of costs is strongly simple and homogenous of degree one in connection costs. Consequently linear strongly simple rules are continuous in cost structures.

\section{Proof of Theorem 1}

We leave it to the reader to check that simple allocation rules on $\mathscr{U}_{U}$ satisfy UII and NI. Consequently we focus on the converse claims and consider cost allocation problems with identical sets of locations.

The proof that allocation rules satisfying UII and NI are simple on $\mathscr{U}_{U}$ consists of a preliminary observation, three lemmas and a closing observation.

First, we make the following preliminary observation. Consider a cost allocation problem $(g, P, C)$ and a finite number of pairs of MCCNs and cost structures $\left(g^{1}, C^{1}\right), \ldots,\left(g^{N}, C^{N}\right)$ such that

$$
\begin{aligned}
& g, g^{1} \in \mathscr{M} \mathscr{C} \mathscr{C} \mathscr{N}\left(P, C^{1}\right) \text { and } c_{j k}^{1}=c_{j k} \text { for all } j k \in g . \\
& g^{1}, g^{2} \in \mathscr{M} \mathscr{C} \mathscr{C} \mathscr{N}\left(P, C^{2}\right) \text { and } c_{j k}^{2}=c_{j k}^{1} \text { for all } j k \in g^{1} . \\
& \quad \vdots \\
& g^{N-1}, g^{N} \in \mathscr{M} \mathscr{C} \mathscr{C} \mathscr{N}\left(P, C^{N}\right) \text { and } c_{j k}^{N}=c_{j k}^{N-1} \text { for all } j k \in g^{N-1} .
\end{aligned}
$$

Then $\phi\left(g^{N}, P, C^{N}\right)=\phi(g, P, C)$ according to UII and NI.

Lemma 1 Consider a cost allocation problem $(g, P, C)$. Suppose that there is a location $u$ with $u \in \mathscr{N}$ and $u \notin g$. Then for every pair of connections rs and st in $g$ and all cost structures $C^{\prime}$ with $c_{r s}^{\prime}+c_{s t}^{\prime}=c_{r s}+c_{s t}, c_{j k}^{\prime}=c_{j k}$ for all other connections in $g$ and $c_{j k}^{\prime}=v(g, c)$ for all other connections, $\phi(g, P, C)=\phi\left(g, P, C^{\prime}\right)$.

Proof: Without loss of generality assume that $c_{r s}^{\prime}<c_{r s}$ and $c_{s t}^{\prime}>c_{s t}$.

In case removing $r s$ and $s t$ from $g$ and adding $r t$ to $g$ result in a graph, which is a $\mathrm{CN}$ consider the following three steps.

Step 1: Define $g^{1}$ by $g^{1}=g$ and define $C^{1}$ by

$$
c_{j k}^{1}=\left\{\begin{array}{cl}
c_{j k} & \text { for } j k \in g \\
v(g, c) & \text { for all other connections. }
\end{array}\right.
$$


Then $g^{1} \in \mathscr{M} \mathscr{C} \mathscr{C} \mathscr{N}\left(P, C^{1}\right)$ and $c_{j k}^{1}=c_{j k}$ for all $j k \in g^{1}$. Therefore $\phi\left(g^{1}, P, C^{1}\right)=\phi(g, P, C)$ according to UII.

Step 2: Define $g^{2}$ by removing $r s$ and $s t$ from $g^{1}$ and adding $r t$. Then $g^{2}$ is a CN. Define $C^{2}$ by

$$
c_{j k}^{2}=\left\{\begin{array}{cl}
c_{r s}^{1}+c_{s t}^{1} & \text { for } j k=r t \\
c_{j k}^{1} & \text { for all other connections. }
\end{array}\right.
$$

Then $g^{1}, g^{2} \in \mathscr{M} \mathscr{C} \mathscr{C} \mathscr{N}\left(P, C^{2}\right)$ and $c_{j k}^{2}=c_{j k}^{1}$ for all $j k \in g^{1}$. Therefore $\phi\left(g^{2}, P, C^{2}\right)=\phi\left(g^{1}, P, C^{1}\right)$ according to UII and NI.

Step 3: Define $g^{3}$ by removing $r t$ from $g^{2}$ and adding $r s$ and $s t$ to $g^{2}$. Then $g^{3}$ is a $\mathrm{CN}$. Indeed $g^{3}=g^{1}$. Define $C^{3}$ by

$$
c_{j k}^{3}= \begin{cases}c_{r s}^{\prime} & \text { for } j k=r s \\ c_{s t}^{\prime} & \text { for } j k=s t \\ c_{j k}^{2} & \text { for all other connections. }\end{cases}
$$

Then $g^{2}, g^{3} \in \mathscr{M} \mathscr{C} \mathscr{C} \mathscr{N}\left(P, C^{3}\right)$ and $c_{j k}^{3}=c_{j k}^{2}$ for all $j k \in g^{2}$. Therefore $\phi\left(g^{3}, P, C^{3}\right)=\phi\left(g^{2}, P, C^{2}\right)$ according to UII and NI. Moreover $c_{r s}^{3}=c_{r s}^{\prime}$ and $c_{s t}^{3}=c_{s t}^{\prime}$.

In case removing $r s$ and $s t$ from $g$ and adding $r t$ to $g$ result in a graph, which is not a $\mathrm{CN}$, consider the following four steps.

Step 1: Define $g^{1}$ by $g^{1}=g$ and define $C^{1}$ by

$$
c_{j k}^{1}=\left\{\begin{array}{cl}
c_{j k} & \text { for } j k \in g \\
v(g, c) & \text { for all other connections. }
\end{array}\right.
$$

Then $g, g^{1} \in \mathscr{M} \mathscr{C} \mathscr{C} \mathscr{N}\left(P, C^{1}\right)$ and $c_{j k}^{1}=c_{j k}$ for all $j k \in g$. Therefore $\phi\left(g^{1}, P, C^{1}\right)=\phi(g, P, C)$ according to UII.

Step 2: Define $g^{2}$ by removing $r s$ and $s t$ from $g^{1}$ and adding $r u, s u$ and $t u$ to $g^{1}$. Then $g^{2}$ is a CN. Define $C^{2}$ by

$$
c_{j k}^{2}=\left\{\begin{array}{cl}
\max \left\{c_{r s}^{\prime}, c_{r s}^{1}-c_{s t}^{1}\right\} & \text { for } j k=r u \\
c_{r s}^{1}-\max \left\{c_{r s}^{\prime}, c_{r s}^{1}-c_{s t}^{1}\right\} & \text { for } j k=s u \\
c_{s t}^{1} & \text { for } j k=t u \\
c_{j k}^{1} & \text { for all other connections. }
\end{array}\right.
$$

Then $g^{1}, g^{2} \in \mathscr{M} \mathscr{C} \mathscr{C} \mathscr{N}\left(P, C^{2}\right)$ and $c_{j k}^{2}=c_{j k}^{1}$ for all $j k \in g^{1}$. Therefore $\phi\left(g^{2}, P, C^{2}\right)=\phi\left(g^{1}, P, C^{1}\right)$ according to UII and NI. 
Step 3: Define $g^{3}$ by removing $r u, s u$ and $t u$ from $g^{2}$ and adding $r s$ and $s t$ to $g^{2}$. Then $g^{3}$ is a CN. Indeed $g^{3}=g^{1}$. Define $C^{3}$ by

$$
c_{j k}^{3}=\left\{\begin{array}{cl}
c_{r u}^{2} & \text { for } j k=r s \\
c_{s u}^{2}+c_{t u}^{2} & \text { for } j k=s t \\
c_{j k}^{2} & \text { for all other connections. }
\end{array}\right.
$$

Then $g^{2}, g^{3} \in \mathscr{M} \mathscr{C} \mathscr{C} \mathscr{N}\left(P, C^{3}\right)$ and $c_{j k}^{3}=c_{j k}^{2}$ for all $j k \in g^{1}$. Therefore $\phi\left(g^{3}, P, C^{3}\right)=\phi\left(g^{2}, P, C^{2}\right)$ according to UII and NI. Moreover $c_{r s}^{3}<c_{r s}^{1}$ and $c_{s t}^{3}>c_{s t}^{1}$.

Step 4: Repeat steps 2 and 3 until the $c_{r s}^{3}=c_{r s}^{\prime}$ and $c_{s t}^{3}=c_{s t}^{\prime}$.

Lemma 2 Consider a cost allocation problem $(g, P, C)$. For every pair of connections $r s$ and $r^{\prime} s^{\prime}$ in $g$, where there is no path between $r$ and $r^{\prime}$ in $g$, and all cost structures $C^{\prime}$ with $c_{r s}^{\prime}+c_{r^{\prime} s^{\prime}}^{\prime}=c_{r s}+c_{r^{\prime} s^{\prime}}$ and $c_{j k}^{\prime}=c_{j k}$ for all other connections, $\phi(g, P, C)=\phi\left(g, P, C^{\prime}\right)$.

Proof: Without loss of generality assume that $c_{r s}^{\prime}<c_{r s}$ and $c_{r^{\prime} s^{\prime}}^{\prime}>c_{r^{\prime} s^{\prime}}$.

Step 1: Define $g^{1}$ by $g^{1}=g$ and define $C^{1}$ by

$$
c_{j k}^{1}=\left\{\begin{array}{cl}
c_{j k} & \text { for } j k \in g \\
v(g, c) & \text { for all other connections. }
\end{array}\right.
$$

Then $g, g^{1} \in \mathscr{M} \mathscr{C} \mathscr{C} \mathscr{N}\left(P, C^{1}\right)$ and $c_{j k}^{1}=c_{j k}$ for all $j k \in g$. Therefore $\phi\left(g^{1}, P, C^{1}\right)=\phi(g, P, C)$ according to UII.

Step 2: Define $g^{2}$ by removing $r s$ and $r^{\prime} s^{\prime}$ from $g^{1}$ and adding $r r^{\prime}, r^{\prime} s$ and $s s^{\prime}$ to $g^{1}$. Then $g^{2}$ is a $\mathrm{CN}$. Define $C^{2}$ by

$$
c_{j k}^{2}=\left\{\begin{array}{cl}
\max \left\{c_{r s}^{\prime}, \frac{c_{r s}^{1}}{2}\right\} & \text { for } j k=r r^{\prime} \\
c_{r s}^{1}-\max \left\{c_{r s}^{\prime}, \frac{c_{r s}^{1}}{2}\right\} & \text { for } j k=r^{\prime} s \\
c_{r^{\prime} s^{\prime}} & \text { for } j k=s s^{\prime} \\
c_{j k}^{1} & \text { for all other connections. }
\end{array}\right.
$$

Then $g^{1}, g^{2} \in \mathscr{M} \mathscr{C} \mathscr{C} \mathscr{N}\left(P, C^{2}\right)$ and $c_{j k}^{2}=c_{j k}^{1}$ for all $j k \in g^{1}$. Therefore $\phi\left(g^{2}, P, C^{2}\right)=\phi\left(g^{1}, P, C^{1}\right)$ according to UII and NI. 
Step 3: Defined $g^{3}$ by removing $r r^{\prime}, r^{\prime} s$ and $s s^{\prime}$ from $g^{2}$ and adding $r s$ and $r^{\prime} s^{\prime}$ to $g^{2}$, so $g^{3}=g$. Define $C^{3}$ by

$$
c_{j k}^{3}=\left\{\begin{array}{cl}
c_{r r^{\prime}}^{2} & \text { for } j k=r s \\
c_{r^{\prime} s}^{2}+c_{s s^{\prime}} & \text { for } j k=r^{\prime} s^{\prime} \\
c_{j k}^{2} & \text { for all other connections. }
\end{array}\right.
$$

Then $g^{2}, g^{3} \in \mathscr{M} \mathscr{C} \mathscr{C} \mathscr{N}\left(P, C^{3}\right)$ and $c_{j k}^{3}=c_{j k}^{2}$ for all $j k \in g^{1}$. Therefore $\phi\left(g^{3}, P, C^{3}\right)=\phi\left(g^{2}, P, C^{2}\right)$ according to UII and NI.

Step 4: Repeat steps 2 and 3 until the $c_{r s}^{3}=c_{r s}^{\prime}$ and $c_{r^{\prime} s^{\prime}}^{3}=c_{r^{\prime} s^{\prime}}^{\prime}$.

Lemma 3 Consider two cost allocation problems $(g, P, C)$ and $(h, P, D)$ with $v(g, c)=v(h, d)$.

Then $\phi(g, P, C)=\phi(h, P, D)$.

Proof: First the cost allocation problem $(g, P, C)$ is transformed into $\left(g^{2}, P, C^{2}\right)$ with $\phi\left(g^{2}, P, C^{2}\right)=$ $\phi(g, P, C)$ such that there is a location $u$ with $u \notin g^{2}$. Consider the following two steps.

Step 1: Define $g^{1}$ by $g^{1}=g$ and define $C^{1}$ by

$$
c_{j k}^{1}=\left\{\begin{array}{cl}
c_{j k} & \text { for } j k \in g \\
v(g, c) & \text { for all other connections. }
\end{array}\right.
$$

Then $g^{1} \in \mathscr{M} \mathscr{C} \mathscr{C} \mathscr{N}\left(P, C^{1}\right)$ and $c_{j k}^{1}=c_{j k}$ for all $j k \in g$. Therefore $\phi\left(g^{1}, P, C^{1}\right)=\phi(g, P, C)$ according to UII.

Step 2: Define $g^{2}$ by removing $r u$ and $s u$ from $g^{1}$ and adding $r s$ to $g^{1}$ as well as replacing all other connections to $u$ in $g^{1}$ with connections to $r$. Then $g^{2}$ is a CN. Define $C^{2}$ by

$$
c_{j k}^{2}=\left\{\begin{array}{cl}
c_{r u}^{1}+c_{s u}^{1} & \text { for } j k=r s \\
c_{j u}^{1} & \text { for all } j \text { with } j u \in g \text { and } k=r \\
c_{j k}^{1} & \text { for all other connections. }
\end{array}\right.
$$

Then $g^{1}, g^{2} \in \mathscr{M} \mathscr{C} \mathscr{C} \mathscr{N}\left(P, C^{2}\right)$ and $c_{j k}^{2}=c_{j k}^{1}$ for all $j k \in g^{1}$. Therefore $\phi\left(g^{2}, P, C^{2}\right)=\phi\left(g^{1}, P, C^{1}\right)$ according to UII and NI.

Let $M$ be a subset of $\mathscr{M}$ such that

- If $h$ is a $\mathrm{CN}$ for $\left(a_{i}, b_{i}\right)_{i \in M}$, then $h$ is a $\mathrm{CN}$ for $P$.

- For all $i \in M$ there exists a CN $h$ for $\left(a_{i^{\prime}}, b_{i^{\prime}}\right)_{i^{\prime} \in M \backslash\{i\}}$ such that $h$ is not a CN for $P$. 
Second $\left(g^{2}, P, C^{2}\right)$ is transformed into $(\tilde{g}, P, \tilde{C})$ with $\phi(\tilde{g}, P, \tilde{C})=\phi\left(g^{2}, P, C^{2}\right)$ such that $\tilde{g}=$ $\cup_{i \in M}\left\{a_{i}, b_{i}\right\}$ and $\tilde{C}$ is arbitrary with $\sum_{i \in M} \tilde{c}_{a_{i} b_{i}}=v(g, c)$ and $\tilde{c}_{j k}=v(g, c)$ for all other connections. Consider the following three steps.

Step 3: Pick $i \in M$ with $a_{i} b_{i} \notin g^{2}$. Then there exists a connection $j^{\prime} k^{\prime}$ in the path $p_{i}\left(g^{2}\right)$ between $a_{i}$ and $b_{i}$ such that $j^{\prime} k^{\prime} \notin\left\{\left(a_{i^{\prime}}, b_{i^{\prime}}\right)_{i^{\prime} \in M}\right\}$. Apply Lemma 1 to move connection costs in the path $p_{i}\left(g^{2}\right)$ such that $c_{j^{\prime} k^{\prime}} \geq \sum_{j k \in p_{i}\left(g^{2}\right) \backslash j^{\prime} k^{\prime}} c_{j k}$. Define $h$ by removing $j^{\prime} k^{\prime}$ from $g^{2}$ and adding $a_{i} b_{i}$ to $g^{2}$. Define $g^{3}$ by removing all connections in $h \backslash \cup_{i^{\prime} \in M} p_{i^{\prime}}(h)$ from $h$. Define $C^{3}$ by

$$
c_{j k}^{3}=\left\{\begin{array}{cl}
c_{j^{\prime} k^{\prime}}^{2}+\sum_{j^{\prime \prime} k^{\prime \prime} \in h \backslash \cup_{i^{\prime} \in M} p_{i^{\prime}}(h)} c_{j^{\prime \prime} k^{\prime \prime}}^{2} & \text { for } j k=a_{i} b_{i} \\
c_{j k}^{2} & \text { for all other connections. }
\end{array}\right.
$$

Then $g^{2}, g^{3} \in \mathscr{M} \mathscr{C} \mathscr{C} \mathscr{N}\left(P, C^{3}\right)$ and $c_{j k}^{3}=c_{j k}^{2}$ for all $j k \in g^{2}$. Therefore $\phi\left(g^{3}, P, C^{3}\right)=\phi\left(g^{2}, P, C^{2}\right)$ according to UII and NI.

Step 4: Repeat step 3 until $a_{i} b_{i} \in g^{3}$ for all $i \in M$ so $g^{3}=\cup_{i \in M}\left\{a_{i}, b_{i}\right\}$.

Step 5: Apply Lemmas 1 and 2 to move cost to an arbitrary $\tilde{C}$ with $\sum_{i \in M} \tilde{c}_{a_{i} b_{i}}=v(g, c)$ and $\tilde{c}_{j k}=v(g, c)$ for all other connections. Then $\tilde{g} \in \mathscr{M} \mathscr{C} \mathscr{C} \mathscr{N}(P, \tilde{C})$ for $\tilde{g}=g^{3}$ and $\phi(\tilde{g}, P, \tilde{C})=$ $\phi\left(g^{3}, P, C^{3}\right)$.

All in all $\phi(g, P, C)=\phi(\tilde{g}, P, \tilde{C})=\phi(h, P, D)$.

We conclude the proof of Theorem 1 for cost allocation problems in $\mathscr{U}_{U}$ with the following observation: according to Lemma 3 the allocation of costs depends on the connection structure $P$, the set of locations and the total cost of the MCCN $v(g, c)$ and no other feature of the cost allocation problem $(g, P, C)$. Therefore the allocation rule is simple.

\section{Implementation}

In the present section we consider a network formation game with $m$ agents and a central planner. All the agents and the planner know the set of finitely many locations $\mathscr{N} \subset \mathscr{L}$ with $|\mathscr{N}| \geq 3$ to ensure some connections are unused. The agents have private information about their connection demands and know all connection costs. The planner is ignorant, so the planner has to estimate connection costs based on connection costs reported by agents. Therefore the rules of the game will consist of an allocation rule and a cost estimation rule $\tau: \mathbb{R}_{++}^{m} \rightarrow \mathbb{R}_{++}$that for every connection $j k$ maps collections of $m$ individual cost reports $\left(\sigma_{1}^{j k}, \ldots, \sigma_{m}^{j k}\right)$ to estimated connection costs $c_{j k}^{e}=\tau\left(\sigma_{1}^{j k}, \ldots, \sigma_{m}^{j k}\right)$. The agents need to ensure that their individual connection demands are satisfied and want to minimize their 
individual cost shares. The planner wants to minimize total cost subject to the constraint that all connection demands are satisfied.

\section{The game}

The network formation game has four stages:

(1) A planner announces a cost allocation rule and a cost estimation rule.

(2) Every agent reports her connection demand and all connection costs.

(3) The planner selects a MCCN based on reported connection demands and estimated connection costs.

(4) The planner allocates true costs of the selected network.

The connection cost reports of the agents are used by the planner to estimate the cost structures. Agents can misreport both their individual connection demands $\left(a_{i}, b_{i}\right)$ and the cost structure $C$. Misreporting influences the estimates of connection demands and costs. Perceived connection demands and estimated connection costs influence the selection of MCCNs. However, true costs rather than estimated costs of the selected MCCN are observed. Therefore true costs rather than estimated costs of the selected MCCN are allocated among agents. Hence misreporting has an indirect influence rather than direct influence on cost shares.

Since the planner selects a MCCN based on estimated connection costs, the selected network need not be a MCCN for the true connection costs. Therefore the set of cost allocation problems has to be extended and cost allocation rules have to be defined on the extended set of cost allocation problems: (1) a cost allocation problem $(g, P, C)$ is a $\mathrm{CN} g$ and a connection problem $(P, C)$ such that $g$ contains no redundant connections; (2) $\mathscr{V}$ is the set of cost allocation problems; and, (3) $\Phi: \mathscr{V} \rightarrow \mathbb{R}_{+}^{m}$ with $\sum_{i \in \mathscr{M}} \Phi_{i}(g, P, C)=v(g, c)$ is an allocation rule. Note $\mathscr{U} \subset \mathscr{V}$ and $\left.\Phi\right|_{\mathscr{U}}$ is an allocation rule on $\mathscr{U}$.

Formally, the rules of the game consist of an allocation rule $\Phi$ and a connection cost estimation rule $\tau: \mathbb{R}_{++}^{m} \rightarrow \mathbb{R}_{++}$. Let $\sigma=\left(\sigma_{1}, \ldots, \sigma_{m}\right)$, where $\sigma_{i}=\left(\sigma_{i}^{j k}\right)_{j k \in \mathscr{N}^{2}}$ for all $i$ with $\sigma_{i}^{j k}>0$ and $\sigma_{i}^{j k}=\sigma_{i}^{k j}$ for all $j \neq k$ and $\sigma_{i}^{j k}=0$ for all $j=k$, be a collection of individual cost reports. The connection cost estimation rule is supposed to take values between the minimum and maximum reported costs

$$
\tau\left(\sigma_{1}^{j k}, \ldots, \sigma_{m}^{j k}\right) \in\left[\min _{i}\left\{\sigma_{i}^{j k}\right\}, \max _{i}\left\{\sigma_{i}^{j k}\right\}\right] .
$$

An connection cost estimation rule is sensitive provided

$$
\tau\left(\mu_{i}^{j k}, \sigma_{-i}^{j k}\right)>\tau\left(v_{i}^{j k}, \sigma_{-i}^{j k}\right)
$$


for all $\mu_{i}^{j k}$ and $v_{i}^{j k}$ with $\mu_{i}^{j k}>v_{i}^{j k}$ and $\sigma_{-i}^{j k}=\left(\sigma_{i^{\prime}}^{j k}\right)_{i^{\prime} \neq i}$ with $\sigma_{i^{\prime}}^{j k}=\sigma_{i^{\prime \prime}}^{j k}$ for all $i^{\prime}, i^{\prime \prime} \neq i$. An estimation rule is unbounded provided for every $i$ and all $\sigma_{-i}^{j k}$,

$$
\lim _{\sigma_{i}^{j k} \rightarrow 0} \tau\left(\sigma_{i}^{j k}, \sigma_{-i}^{j k}\right)=0 \text { and } \lim _{\sigma_{i}^{j k} \rightarrow \infty} \tau\left(\sigma_{i}^{j k}, \sigma_{-i}^{j k}\right)=\infty .
$$

The geometric average is sensitive and unbounded. Estimation rules like the arithmetic and geometric averages as well as nontrivial convex combinations of the minimum report and the maximum report are sensitive. The minimum report is not sensitive or unbounded, but it is downward sensitive in the sense that it is sensitive for $\mu_{i}^{j k}, v_{i}^{j k} \leq \sigma_{i^{\prime}}^{j k}$ for all $i^{\prime} \neq i$ and downward unbounded in the sense that

$$
\lim _{\sigma_{i}^{j k} \rightarrow 0} \tau\left(\sigma_{i}^{j k}, \sigma_{-i}^{j k}\right)=0
$$

The maximum report is not sensitive, but it is upward sensitive in the sense that it is sensitive for $\mu_{i}^{j k}, v_{i}^{j k} \geq \sigma_{i^{\prime}}^{j k}$ for all $i^{\prime} \neq i$ and upward unbounded in the sense that

$$
\lim _{\sigma_{i}^{j k} \rightarrow \infty} \tau\left(\sigma_{i}^{j k}, \sigma_{-i}^{j k}\right)=\infty
$$

The median report is neither sensitive nor unbounded. In general with estimation rules being neither sensitive nor unbounded individual agents are not able to influence estimates of connection costs. Therefore all reports are equally good for every individual agent.

The payoff of every agent is minus her cost share in case her connection demand is met and $-\infty$ otherwise. Every agent reports her connection demand and all connection costs $s_{i}=\left(\omega_{i}, \sigma_{i}\right)$ where $\omega_{i}=\left(\alpha_{i}, \beta_{i}\right) \in \mathscr{N}^{2}$. Hence the strategy set of every agent is $\mathscr{N}^{2} \times \mathscr{C}$ where $\mathscr{C}$ is the set of all cost structures

$$
\mathscr{C}=\left\{\left(\sigma^{j k}\right)_{j k \in \mathscr{N}^{2}} \in \mathbb{R}_{+}^{\mathscr{N}^{2}} \mid \begin{array}{l}
\sigma_{i}^{j k}>0 \text { and } \sigma_{i}^{j k}=\sigma_{i}^{k j} \text { for all } j \neq k \\
\sigma_{i}^{j k}=0 \text { for all } j=k
\end{array}\right\} .
$$

Let $\omega$, where $\omega=\left(\omega_{1}, \ldots, \omega_{m}\right)$ and $\omega_{i}=\left(\alpha_{i}, \beta_{i}\right) \in \mathscr{N}^{2}$ for all $i$, be a collection of individual reports on connection demands. Then the planner uses these reports $\omega$ as connection demands. For a collection of individual cost reports $\sigma$ the estimated connection costs are $c^{e}(\sigma)$, where $c_{j k}^{e}(\sigma)=\tau\left(\sigma_{i}^{j k}, \ldots, \sigma_{m}^{j k}\right)$ for all $j k$. The planner allocates observed costs. Thus let $\rho\left(g, c, c^{e}\right): \mathscr{N}^{2} \rightarrow \mathbb{R}_{+}$be defined by

$$
\rho_{j k}\left(g, c, c^{e}\right)=\left\{\begin{array}{cl}
c_{j k} & \text { for all } j k \in g \\
c_{j k}^{e} & \text { for all } j k \notin g
\end{array}\right.
$$

Therefore for every network $g$ in $\mathscr{M} \mathscr{C} \mathscr{C} \mathscr{N}\left(\omega, c^{e}(\sigma)\right)$ the allocation of observed costs is $\Phi\left(g, \omega, \rho\left(g, c, c^{e}(\sigma)\right)\right)$. The planner randomly selects a network $g$ in $\mathscr{M} \mathscr{C} \mathscr{C} \mathscr{N}\left(\omega, c^{e}(\sigma)\right)$ 
with all networks being equally likely. Hence for fixed collections of individual reports $(\omega, \sigma)$ the expected allocation of costs is

$$
\mathrm{E} \Phi\left(\omega, c, c^{e}(\sigma)\right)=\frac{1}{\left|\mathscr{M} \mathscr{C} \mathscr{C} \mathscr{N}\left(\omega, c^{e}(\sigma)\right)\right|} \sum_{g \in \mathscr{M} \mathscr{C} \mathscr{N}\left(\omega, c^{e}(\sigma)\right)} \Phi\left(g, \omega, \rho\left(g, c, c^{e}(\sigma)\right)\right) .
$$

Agents can choose their reports strategically.

\section{Equilibrium}

The notion of equilibrium is Nash equilibrium.

Definition 5 A Nash equilibrium is a collection of individual reports $(\bar{\omega}, \bar{\sigma})$ such that for every agent $i$ and all reports $\left(\omega_{i}, \sigma_{i}\right)$ with $p_{i}(g) \neq \emptyset$ for all $g \in \mathscr{M} \mathscr{C} \mathscr{C} \mathscr{N}\left(\omega_{i}, \bar{\omega}_{-i}, c^{e}\left(\sigma_{i}, \bar{\sigma}_{-i}\right)\right)$,

$$
\mathrm{E} \Phi_{i}\left(\left(\omega_{i}, \bar{\omega}_{-i}\right), c, c^{e}\left(\sigma_{i}, \bar{\sigma}_{-i}\right)\right) \geq \mathrm{E}_{i}\left(\bar{\omega}, c, c^{e}(\bar{\sigma})\right) .
$$

Let $\mathscr{N} \mathscr{E}(P, C, \Phi, \tau)$ be the set of Nash equilibria.

\section{No truth-telling without UII and NI}

In the two observations below we show that if truth-telling is a Nash equilibrium, then the allocation rule satisfies both UII and NI provided the estimation rule is continuous and sensitive. We consider UII rather than SUII because both the agents and the planner know the set of locations is $\mathscr{N}$,

Misreporting connection costs of unused connections influences the cost estimates of unused connections. Moreover if an allocation rule does not satisfy UII, the cost estimates of unused connections influence cost shares. Therefore agents can manipulate their cost shares by misreporting provided UII is not satisfied. Furthermore misreporting need not be revealed because true connection costs of unused connections are unobserved.

Observation 1 Suppose $\Phi$ is continuous in cost structures and $\tau$ is continuous and sensitive. If truth-telling is a Nash equilibrium, then $\Phi$ satisfies UII.

Proof: Suppose a cost allocation rule $\Phi$ does not satisfy UII restricted to cost allocation problems with $\mathscr{N}$ as the set of locations. Then there are a pair cost allocation problems $(g, P, C)$ and $(g, P, D)$ with $\mathscr{N}_{C}=\mathscr{N}_{D}=\mathscr{N}$ and $c_{j k}=d_{j k}$ for all $j k \in g$ and an agent $i$ such that $\Phi_{i}(g, P, C) \neq \Phi_{i}(g, P, D)$. For all $\varepsilon>0$ define two other cost structures $C^{1}$ and $D^{1}$ by

$$
c_{j k}^{1}=\left\{\begin{array}{cl}
c_{j k} & \text { for } j k \in g \\
c_{j k}+\varepsilon & \text { for all other connections }
\end{array}\right.
$$


and

$$
d_{j k}^{1}=\left\{\begin{array}{cl}
d_{j k} & \text { for } j k \in g \\
d_{j k}+\varepsilon & \text { for all other connections. }
\end{array}\right.
$$

Then $g$ is the unique MCCN for connection problems $\left(P, C^{1}\right)$ and $\left(P, D^{1}\right)$ for all $\varepsilon$. For $\varepsilon$ sufficiently small $\Phi_{i}\left(g, P, C^{1}\right) \neq \Phi_{i}\left(g, P, D^{1}\right)$ because $\Phi$ is continuous in cost structures. Without loss of generality suppose $\Phi_{i}\left(g, P, C^{1}\right)>\Phi_{i}\left(g, P, D^{1}\right)$. Let $\bar{\delta} \in[0,1]$ be defined by

$$
\bar{\delta}=\max \left\{\delta \in[0,1] \mid \Phi_{i}\left(g, P,(1-\delta) C^{1}+\delta D^{1}\right)=\Phi_{i}\left(g, P, C^{1}\right)\right\} .
$$

Then $\bar{\delta}<1$ and $\phi_{i}\left(g, P,(1-\delta) C^{1}+\delta D^{1}\right)<\phi_{i}\left(g, P, C^{1}\right)$ for all $\left.\left.\delta \in\right] \bar{\delta}, 1\right]$. For the connection problem $\left(P,(1-\bar{\delta}) C^{1}+\bar{\delta} D^{1}\right)$ if all agents except agent $i$ are telling the truth, then the strategy $\left(\omega_{i}, \sigma_{i}\right)$, where $\omega_{i}=\left(a_{i}, b_{i}\right)$ and $\sigma_{i}$ is such that for some $\left.\left.\delta \in\right] \bar{\delta}, 1\right]$ and all $j k$, $\tau\left(\sigma_{i}^{j k},\left(c_{j k}\right)_{i^{\prime} \neq i}\right)=(1-\delta) c_{j k}^{1}+\delta d_{j k}^{1}$ lowers the cost share of agent $i$. There exists $\sigma^{i}$ such that for some $\delta \in] \bar{\delta}, 1]$ and all $j k, \tau\left(\sigma_{i}^{j k},\left(c_{j k}\right)_{i^{\prime} \neq i}\right)=(1-\delta) c_{j k}^{1}+\delta d_{j k}^{1}$ because $\tau$ is continuous and sensitive.

Misreporting connection costs influences cost estimates and cost estimates influence the set of estimated MCCNs. Moreover if an allocation rule does not satisfy NI, then cost shares depend on the selected MCCN. Therefore agents can manipulate their cost shares by misreporting provided NI is not satisfied. Furthermore misreporting need not be revealed because true connection costs of unused connections are unobserved.

Observation 2 Suppose $\Phi$ is continuous in cost structures and $\tau$ is continuous and upward or downward sensitive. If truth-telling is a Nash equilibrium, then $\Phi$ satisfies NI.

Proof: Suppose a cost allocation rule $\Phi$ does not satisfy NI. Then there is a connection problem $(P, C)$ and an agent $i$ such that

$$
\min _{g \in \mathscr{M} \mathscr{C} \mathscr{C} \mathscr{N}(P, C)} \Phi_{i}(g, P, C)<\max _{h \in \mathscr{M} \mathscr{C} \mathscr{C} N(P, C)} \Phi_{i}(h, P, C) .
$$

Therefore suppose $\Phi_{i}(g, P, C) \leq \Phi_{i}(h, P, C)$ for $g$ and $i$ and all $h \in \mathscr{M} \mathscr{C} \mathscr{C} \mathscr{N}(P, C)$ and $\Phi_{i}(g, P, C)<$ $\Phi_{i}\left(h^{\prime}, P, C\right)$ for $g$ and $i$ and some $h^{\prime} \in \mathscr{M} \mathscr{C} \mathscr{C} \mathscr{N}(P, C)$. For $\varepsilon>0$ define another cost structure $C^{1}$ by

$$
c_{j k}^{1}=\left\{\begin{array}{cl}
c_{j k} & \text { for } j k \in g \\
c_{j k}+\varepsilon & \text { for all other connections }
\end{array}\right.
$$

Then $g$ is the unique MCCN for connection problem $\left(P, C^{1}\right)$ for all $\varepsilon$. For $\varepsilon$ sufficiently small

$$
\Phi_{i}\left(g, P, C^{1}\right)<\frac{1}{|\mathscr{M} \mathscr{C} \mathscr{C} \mathscr{N}(P, C)|} \sum_{h \in \mathscr{M} \mathscr{C} \mathscr{C} N(P, C)} \Phi_{i}(h, P, C)
$$


because $\Phi$ is continuous in cost structures. For the connection problem $(P, C)$ if all agents except agent $i$ are telling the truth, then the strategy $\left(\omega_{i}, \sigma_{i}\right)$, where $\omega_{i}=\left(a_{i}, b_{i}\right)$ and $\sigma_{i}$ is such that $\tau\left(\sigma_{i}^{j k},\left(c_{j k}\right)_{i^{\prime} \neq i}\right)=c_{j k}^{1}$, lowers the expected cost share of agent $i$. There exist $\varepsilon>0$ and $\sigma_{i}$ such that $\tau\left(\sigma_{i}^{j k},\left(c_{j k}\right)_{i^{\prime} \neq i}\right)=c_{j k}^{1}$ because $\tau$ is continuous and upward sensitive. Hence truth-telling is not an equilibrium for $(P, C)$. Alternative, there exist $\varepsilon>0$ and $\sigma_{i}$ such that $\tau\left(\sigma_{i}^{j k},\left(c_{j k}\right)_{i^{\prime} \neq i}\right)=c_{j k}-\varepsilon$ for $j k \in g$ and $\tau\left(\sigma_{i}^{j k},\left(c_{j k}\right)_{i^{\prime} \neq i}\right)=c_{j k}$ for $j k \notin g$ because $\tau$ is continuous and downward sensitive.

\section{Truth-telling}

Both UII and NI are necessary for truthful reporting as shown in Observations 1 and 2. Consequently we focus on simple allocation rules.

Definition 6 An allocation rule $\Phi$ is implementable provided truth-telling is a Nash equilibrium for every connection problem $(P, C) \in \mathscr{U}$.

Let $P_{-i}=\left(a_{i^{\prime}}, b_{i^{\prime}}\right)_{i^{\prime} \neq i}$ be a collection of connection demands for all agents except agent $i$. Then a simple allocation rule is implementable if and only if it is increasing in total costs and the cost share of every agent is independent of her connection demand.

Theorem 2 Suppose an allocation rule $\Phi$ is simple and an estimation rule $\tau$ is continuous and sensitive. Then truth-telling is a Nash equilibrium if and only if $\gamma$ is increasing in total costs and $\gamma_{i}\left(\left(a_{i}, b_{i}\right), P_{-i}, \mathscr{N}, v\right)=\gamma_{i}\left(\left(\alpha_{i}, \beta_{i}\right), P_{-i}, \mathscr{N}, v\right)$ for all $i, P_{-i},\left(a_{i}, b_{i}\right),\left(\alpha_{i}, \beta_{i}\right)$ and $v$.

Proof: It is left to the reader to check that if $\gamma$ is increasing in $v$ and $\gamma\left(\left(a_{i}, b_{i}\right), P_{-i}, \mathscr{N}, v\right)=$ $\gamma\left(\left(\alpha_{i}, \beta_{i}\right), P_{-i}, \mathscr{N}, v\right)$ for all $i, P_{-i},\left(a_{i}, b_{i}\right),\left(\alpha_{i}, \beta_{i}\right)$ and $v$, then truth-telling is a Nash equilibrium.

Suppose $\gamma$ is not increasing in $v$. Then there are $P, v_{L}, v_{H}$ with $v_{H}>v_{L}$ and $i$ such that $\gamma_{i}\left(P, \mathscr{N}, v_{H}\right)<\gamma_{i}\left(P, \mathscr{N}, v_{L}\right)$. Therefore for all $\varepsilon>0$ there is $v_{\varepsilon}$ such that $\gamma_{i}(P, \mathscr{N}, \cdot)$ is not increasing in $v \in\left[v_{\varepsilon}-\varepsilon, v_{\varepsilon}+\varepsilon\right] \cap\left[v_{L}, v_{H}\right]$. Let $\bar{v} \in\left[v_{L}, v_{H}\right]$ be an accumulation point for $\left(v_{\varepsilon}\right)_{\varepsilon>0}$. Then for all $\varepsilon>0$ there are $v^{\prime}, v^{\prime \prime} \in[\bar{v}-\varepsilon, \bar{v}+\varepsilon] \cap\left[v_{L}, v_{H}\right]$ such that $v^{\prime}>v^{\prime \prime}$ and $\gamma_{i}\left(P, \mathscr{N}, v^{\prime}\right)<\gamma_{i}\left(P, \mathscr{N}, v^{\prime \prime}\right)$. Hence for all $\varepsilon>0$ there are $v^{\prime}, v^{\prime \prime} \in[\bar{v}-\varepsilon, \bar{v}+\varepsilon] \cap\left[v_{L}, v_{H}\right]$ such that $v^{\prime} \geq \bar{v} \geq v^{\prime \prime}$ and $\gamma\left(P, \mathscr{N}, v^{\prime}\right)<\gamma(P, \mathscr{N}, \bar{v})$ or $\gamma\left(P, \mathscr{N}, v^{\prime \prime}\right)>\gamma(P, \mathscr{N}, \bar{v})$. Hence there is a sequence $\left(v_{n}\right)_{n \in \mathbb{N}}$ with $v_{n} \in\left[v_{L}, v_{H}\right]$ and $\lim _{n \rightarrow \infty} v_{n}=\bar{v}$ such that $v_{n}>\bar{v}$ for all $n$ and $\gamma\left(P, \mathscr{N}, v_{n}\right)<\gamma(P, \mathscr{N}, \bar{v})$ for all $n$ or $v_{n}<\bar{v}$ for all $n$ and $\gamma\left(P, \mathscr{N}, v_{n}\right)>\gamma(P, \mathscr{N}, \bar{v})$ for all $n$.

Let $g$ be a CN minimizing the number of connections $|g|$. For some $r s \in g$ let $g_{r}\left(g_{s}\right)$ be the component containing $r(s)$ of $g$ without $r s$. For $v_{\min }^{n}=\min \left\{v_{n}, \bar{v}\right\}$ and $v_{\max }^{n}=$ $\max \left\{v_{n}, \bar{v}\right\}$ for all $n$ let a sequence of connection costs $\left(c^{n}\right)_{n \in \mathbb{N}}$ be defined as follows: (1) 
$c_{j k}^{n}=(1 /|g|) v_{\min }^{n}$ for all $j k \in g ;(2.1)$ in case $g_{r}=g_{s}=\emptyset$ pick a location $t \neq r, s$ and add $r t$ and $s t$ to $g$ without $r s$ with location costs $c_{r t}^{n}=c_{s t}^{n}=(1 /|g|) v_{\min }^{n} / 2+\left(v_{\max }^{n}-v_{\min }^{n}\right) / 2 ;(2.2)$ in case $g_{r}=\emptyset$ and $g_{s} \neq \emptyset$ pick a location $t \neq s$ in $g_{s}$ and add $r t$ to $g$ without $r s$ with connection $\operatorname{cost} c_{r t}^{n}=(1 /|g|) v_{\text {min }}^{n}+\left(v_{\text {max }}^{n}-v_{\text {min }}^{n}\right) ;(2.3)$ in case $g_{r} \neq \emptyset$ and $g_{s}=\emptyset$ pick a location $t \neq r$ in $g_{r}$ and add $s t$ to $g$ without $r s$ with connection cost $c_{s t}^{n}=(1 /|g|) v_{\min }^{n}+\left(v_{\max }^{n}-v_{\min }^{n}\right)$; (2.4) in case $g_{r}, g_{s} \neq \emptyset$ pick locations $t \neq r$ in $g_{r}$ and $u \neq s$ in $g_{s}$ with connection cost $c_{t u}^{n}=(1 /|g|) v_{\min }^{n}+\left(v_{\max }^{n}-v_{\min }^{n}\right)$; and, (3) let $c_{j k}^{n}=v_{\max }^{n}$ for all other connections $j k$. Then $g$ is the unique MCCN with $v\left(g, c^{n}\right)=v_{\min }^{n}$ for all $n$ and there is a unique CN with no redundant connections $h$ with $v\left(h, c^{n}\right)=v_{\max }^{n}$.

For the connection problem $\left(P, C^{n}\right)$ if all agents except agent $i$ are telling the truth, then the strategy $\left(\omega_{i}, \sigma_{i}\right)$, where $\omega_{i}=\left(a_{i}, b_{i}\right)$ and $\sigma_{i}$ is such that $\tau\left(\sigma_{i}^{j k},\left(c_{j k}\right)_{i^{\prime} \neq i}\right)=c_{j k}^{n}$ for all $j k \in h$ and $\tau\left(\sigma_{i}^{j k},\left(c_{j k}\right)_{i^{\prime} \neq i}\right)=c_{j k}^{n}+\varepsilon_{n}$ for all $j k \notin h$, lowers the expected cost share of agent $i$ for $\varepsilon_{n}=2\left(v_{\max }^{n}-v_{\min }^{n}\right)$. There is $n \in \mathbb{N}$ and $\sigma_{i}$ such that $\tau\left(\sigma_{i}^{j k},\left(c_{j k}\right)_{i^{\prime} \neq i}\right)=c_{j k}^{n}$ for all $j k \in h$ and $\tau\left(\sigma_{i}^{j k},\left(c_{j k}\right)_{i^{\prime} \neq i}\right)=c_{j k}^{n}+\varepsilon_{n}$ for all $j k \notin h$ because $\tau$ is continuous and sensitive. Hence truth-telling is not an equilibrium for $\left(P, C^{n}\right)$ for some $n$.

Suppose $\gamma_{i}\left(\left(a_{i}, b_{i}\right), P_{-i}, \mathscr{N}, v\right)>\gamma_{i}\left(\left(\alpha_{i}, \beta_{i}\right), P_{-i}, \mathscr{N}, v\right)$ for some $i, P_{-i},\left(a_{i}, b_{i}\right),\left(\alpha_{i}, \beta_{i}\right)$ and $v>0$. Consider connection costs $c$ such that for some $i^{\prime} \neq i$ a path between $a_{i^{\prime}}$ and $b_{i^{\prime}}$ going through all locations is the unique MCCN and the cost of the path is $v$. Suppose the true connection structure is $\left(\left(a_{i}, b_{i}\right), P_{-i}\right)$. Then agent $i$ can lower her cost share by changing her strategy from $\left(a_{i}, b_{i}\right)$ to $\left(\alpha_{i}, \beta_{i}\right)$. Therefore truth-telling is not a Nash equilibrium.

Recall that the price of stability $P_{S}(\Phi, \tau) \geq 1$ is the ratio between the cost of the cheapest Nash equilibrium and the cost of a MCCN

$$
P_{S}(\Phi, \tau)=\sup _{(g, P, C) \in \mathscr{U}(\omega, \sigma) \in \mathscr{N} \mathscr{E}(P, C, \Phi, \tau)} \frac{\sum_{i} \mathrm{E} \Phi_{i}\left(\omega, c, c^{e}(\sigma)\right)}{v(g, c)} .
$$

Theorem 2 implies the price of stability is one provided the allocation rule is simple and satisfies the assumption in the theorem.

If an allocation rule is simple and increasing in total cost, then it is continuous in total costs.

Observation 3 Suppose an allocation rule $\Phi$ is simple and $\gamma$ is increasing in total costs. Then $\gamma$ is continuous in total costs.

Proof: Suppose $\gamma$ is not continuous in $v$. Then there are $P, \bar{v}>0$ and $\left(v_{n}\right)_{n \in \mathbb{N}}$ with $\lim _{n \rightarrow \infty} v_{n}=$ $v$ such that $\liminf _{n \rightarrow \infty} \gamma_{i}\left(P, \mathscr{N}, v_{n}\right) \neq \gamma(P, \mathscr{N}, \bar{v})$ or $\limsup _{n \rightarrow \infty} \gamma_{i}\left(P, \mathscr{N}, v_{n}\right) \neq \gamma(P, \mathscr{N}, \bar{v})$. By considering a subsequence it can be assumed that $v_{n}<\bar{v}$ for all $n$ or $v_{n}>\bar{v}$ for all $n$. In case $v_{n}<\bar{v}$ for all $n, \liminf _{n \rightarrow \infty} \gamma_{i}\left(P, \mathscr{N}, v_{n}\right)<\gamma_{i}(P, \mathscr{N}, \bar{v})$ because $\gamma_{i}$ is increasing in total costs. Since $\sum_{i \in \mathscr{M}} \gamma_{i}(P, \mathscr{N}, v)=v$ for all $P$ and $v$, there is $i^{\prime}$ and $n$ such that 
$\gamma_{i^{\prime}}\left(P, \mathscr{N}, v_{n}\right)>\gamma_{i^{\prime}}(P, \mathscr{N}, \bar{v})$ contradicting $\gamma_{i^{\prime}}$ is increasing in total costs. In case $v_{n}>\bar{v}$ for all $n, \limsup _{n \rightarrow \infty} \gamma_{i}\left(P, \mathscr{N}, v_{n}\right)>\gamma_{i}(P, \mathscr{N}, \bar{v})$ because $\gamma_{i}$ is increasing in total costs. Since $\sum_{i \in \mathscr{M}} \gamma_{i}(P, \mathscr{N}, v)=v$ for all $P$ and $v$, there is $i^{\prime}$ and $n$ such that $\gamma_{i^{\prime}}\left(P, \mathscr{N}, v_{n}\right)<\gamma_{i^{\prime}}(P, \mathscr{N}, \bar{v})$ contradicting $\gamma_{i^{\prime}}$ is increasing in total costs.

A linear simple allocation rule is implementable if and only if the cost share of every agent is independent of her connection demand.

Corollary 4 Suppose an allocation rule $\Phi$ is linear simple. Then truth-telling is a Nash equilibrium if and only if $\Gamma_{i}\left(\left(a_{i}, b_{i}\right), P_{-i}, \mathscr{N}\right)=\Gamma_{i}\left(\left(\alpha_{i}, \beta_{i}\right), P_{-i}, \mathscr{N}\right)$ for all $i, P_{-i},\left(a_{i}, b_{i}\right)$ and $\left(\alpha_{i}, \beta_{i}\right)$.

Proof: It is left to the reader to check that if $\Gamma_{i}\left(\left(a_{i}, b_{i}\right), P_{-i}, \mathscr{N}\right)=\Gamma_{i}\left(\left(\alpha_{i}, \beta_{i}\right), P_{-i}, \mathscr{N}\right)$ for all $i, P_{-i},\left(a_{i}, b_{i}\right)$ and $\left(\alpha_{i}, \beta_{i}\right)$, then truth-telling is a Nash equilibrium.

Suppose $\Gamma_{i}\left(\left(a_{i}, b_{i}\right), P_{-i}, \mathscr{N}\right)>\Gamma_{i}\left(\left(\alpha_{i}, \beta_{i}\right), P_{-i}, \mathscr{N}\right)$ for some $i, P_{-i},\left(a_{i}, b_{i}\right),\left(\alpha_{i}, \beta_{i}\right)$. Consider connection costs $c$ such that for some $i^{\prime} \neq i$ a path between $a_{i^{\prime}}$ and $b_{i^{\prime}}$ going through all locations is the unique MCCN. Suppose the true connection structure is $\left(\left(a_{i}, b_{i}\right), P_{-i}\right)$. Then agent $i$ can lower her cost share by changing her strategy from $\left(a_{i}, b_{i}\right)$ to $\left(\alpha_{i}, \beta_{i}\right)$. Therefore truth-telling is not a Nash equilibrium.

\section{A less ignorant planner}

We consider the consequences of: connection demands being known by the planner; and, connection costs being known by the planner.

Theorem 3 Consider the set of connection problems $(P, C)$.

(1) Assume connection demands are known by the planner. For all connection problems $(P, C)$ and all rules of the game $(\Phi, \tau)$, where $\Phi$ is simple and $\tau$ is downward or upward unbounded,

$$
\mathscr{M} \mathscr{C} \mathscr{C} \mathscr{N}\left(P, C^{e}(\bar{\sigma})\right) \subset \mathscr{M} \mathscr{C} \mathscr{C} N(P, C)
$$

for all $\bar{\sigma} \in \mathscr{N} \mathscr{E}(P, C, \Phi, \tau)$.

(2) Assume connection costs are known by the planner. There are connection problems $(P, C)$ such that for all rules of the game $(\Phi, \tau)$, where $\Phi$ is simple,

$$
\mathscr{M} \mathscr{C} \mathscr{C} \mathscr{N}\left(P^{e}(\bar{\omega}), C\right) \cap \mathscr{M} \mathscr{C} \mathscr{C} \mathscr{N}(P, C)=\emptyset
$$

for some $\bar{\omega} \in \mathscr{N} \mathscr{E}(P, C, \Phi, \tau)$. 
Proof: “Known connection demands” Suppose

$\mathscr{M} \mathscr{C} \mathscr{C} \mathscr{N}\left(P, C^{e}(\bar{\sigma})\right) \not \subset \mathscr{M} \mathscr{C} \mathscr{C} \mathscr{N}(P, C)$.

Then there is CN $g$ with $g \in \mathscr{M} \mathscr{C} \mathscr{C} \mathscr{N}\left(P, C^{e}(\bar{\sigma})\right)$ and $g \notin \mathscr{M} \mathscr{C} \mathscr{C} \mathscr{N}(P, C)$ so $v(g, c)>v(h, c)$ for all $h \in \mathscr{M} \mathscr{C} \mathscr{C} \mathscr{N}(P, C)$. Therefore there is an agent $i$ such that

$$
\frac{1}{\left|\mathscr{M} \mathscr{C} \mathscr{C} \mathscr{N}\left(P, C^{e}(\overline{\boldsymbol{\sigma}})\right)\right|} \sum_{g \in \mathscr{M} \mathscr{C} \mathscr{C} N\left(P, C^{e}(\bar{\sigma})\right)} \phi_{i}(g, P, C)>\frac{1}{|\mathscr{M} \mathscr{C} \mathscr{C} \mathscr{N}(P, C)|} \sum_{h \in \mathscr{M} \mathscr{C} \mathscr{N}(P, C)} \phi_{i}(h, P, C)
$$

because $v(g, c) \geq v(h, c)$ for all $g \in \mathscr{M} \mathscr{C} \mathscr{C} \mathscr{N}\left(P, C^{e}(\bar{\sigma})\right)$ and $h \in \mathscr{M} \mathscr{C} \mathscr{C} \mathscr{N}(P, C)$.

If $\tau$ is downward unbounded there is a sequence of strategies $\left(\sigma_{i n}\right)_{n \in \mathbb{N}}$ for agent $i$ such that $\lim _{n \rightarrow \infty} \tau\left(\sigma_{i n}^{j k}, \bar{\sigma}_{-i}^{j k}\right)=0$ for some $h \in \mathscr{M} \mathscr{C} \mathscr{C} \mathscr{N}(P, C)$ and all $j k \in h$. If $\tau$ is upward unbounded there is a sequence of strategies $\left(\sigma_{i n}\right)_{n \in \mathbb{N}}$ such that $\lim _{n \rightarrow \infty} \tau\left(\sigma_{i n}^{j k}, \bar{\sigma}_{-i}^{j k}\right)=\infty$ for some $h \in \mathscr{M} \mathscr{C} \mathscr{C} \mathscr{N}(P, C)$ and all $j k \notin h$. Thus $\bar{\sigma}$ is not a Nash equilibrium.

"Known connection costs" Consider an example with four agents $i \in\{1,2,3,4\}$ and four locations $j \in\{1,2,3,4\}$. Suppose the connection demands are $\left(a_{i}, b_{i}\right)=(2,3)$ for all $i$ and the cost structure is $c_{23}=1, c_{12}=c_{34}=\varepsilon$ and $c_{j k}=1+\varepsilon$ for all other $j k \notin\{12,23,34\}$ where $\varepsilon>0$. Then the unique MCCN is $g=23$ with $v(g, c)=1$. Let the allocation rule be any simple allocation rule satisfying the assumption in Theorem 2. Suppose agents $i \in$ $\{1,2\}$ reports their connection demands as $\left(\alpha_{i}, \beta_{i}\right)=(1,3)$ and agents $i \in\{3,4\}$ reports their connection demands as $\left(\alpha_{i}, \beta_{i}\right)=(2,4)$. Based on the reports the network $h=12,23,34$ is selected with $v(h, c)=1+2 \varepsilon$. Moreover it is not possible for any of the four agents to bring down her cost share by changing her report.

Recall that the price of anarchy $P_{A}(\Phi, \tau) \geq 1$ is the ratio between the cost of the most expensive Nash equilibrium and the cost of a MCCN

$$
P_{S}(\Phi, \tau)=\sup _{(g, P, C) \in \mathscr{U}(\omega, \sigma) \in \mathscr{N} \mathscr{E}(P, C, \Phi, \tau)} \frac{\sum_{i} \mathrm{E} \Phi_{i}\left(\omega, c, c^{e}(\sigma)\right)}{v(g, c)} .
$$

In case connection demands are known as in (1) in Theorem 3 and the allocation rule is increasing in total cost ensuring existence of Nash equilibria according to Theorem 2, the price of anarchy drops to 1. In case connection costs are known as in (2) in Theorem 3 and the cost share of every agent is independent of her connection demand ensuring existence of Nash equilibria according to Theorem 2, the price of anarchy is unbounded. Indeed the proof of (2) in Theorem 3 is an example where the cost of anarchy is $1+2 \varepsilon$ where $\varepsilon>0$ is arbitrary. 


\section{Implementation with incomplete information}

For every agent, information about connection costs and connection demands of other agents can be incomplete: for example, they know their own connection demand and connection costs but only have vague ideas about connection costs and connection demands of others. As discussed in Jackson (1991) incomplete information is naturally modeled within a Bayesian setting.

All agents and the planner know the set of finitely many locations $\mathscr{N} \subset \mathscr{L}$. For agent $i$ let $\mathscr{P}_{i} \subset \mathscr{N} \times \mathscr{N}$ be a set of possible connection demands. Let $\mathscr{P}=\prod_{i} \mathscr{P}_{i}$. Let $\mathscr{C}=$ $\left\{C_{1}, \ldots, C_{\ell}\right\}$ be a finite set of possible cost structures known by all agents. Every agent $i$ has a probability measure on the set of states $\mu_{i}: \mathscr{P} \times \mathscr{C} \rightarrow[0,1]$ with $\mu_{i}(P, C)>0$ for all $(P, C) \in \mathscr{P} \times \mathscr{C}$. In addition every agent $i$ has a signal from the set of possible states to subsets of that set $\delta_{i}: \mathscr{P} \times \mathscr{C} \rightarrow 2^{\mathscr{P} \times \mathscr{C}}$ where $\delta_{i}(P, C)$ is the private information of agent $i$ at $(P, C)$. For signals it is assumed that: (1) every agent knows her own connection demand; (2) if the state is $(P, C)$, then $(P, C)$ is part of the private information at $(P, C) ;(3)\left(P^{\prime}, C^{\prime}\right)$ is part of the private information at $(P, C)$ if and only if $(P, C)$ is part of the private information at $\left(P^{\prime}, C^{\prime}\right)$; and, (4) the intersection of all private information sets consists of the true state. Formally: (1) $\operatorname{pr}_{\mathscr{P}_{i}} \delta_{i}(P, C)=\left(a_{i}, b_{i}\right)$ for $P=\left(a_{i^{\prime}}, b_{i^{\prime}}\right)_{i^{\prime}}$ where $\operatorname{pr}_{\mathscr{P}_{i}}$ is the projection on $\mathscr{P}_{i}$; (2) $(P, C) \in \delta_{i}(P, C) ; 3 .(P, C) \in \delta_{i}\left(P^{\prime}, C^{\prime}\right)$ if and only if $\left(P^{\prime}, C^{\prime}\right) \in \delta_{i}(P, C)$; and $4 . \cap_{i} \delta_{i}(P, C)=$ $(P, C)$.

A connection problem with incomplete information consists of a set of agents, a set of possible states and a list of individual signals and probability measures, $\left(\mathscr{N}, \mathscr{P} \times \mathscr{C},\left(\delta_{i}, \mu_{i}\right)_{i}\right)$. The planner is ignorant in the sense that she knows neither the set of possible states nor the individual lists of signals and probablility measures.

Let $\mathscr{K}$ be the set of all cost structures so $\mathscr{C} \subset \mathscr{K}$. Let $\mathscr{F}$ be the set of all finite subsets of $\mathscr{K}$. The planner chooses a mechanism that consists of an announcement set for every agent, a cost allocation rule and an estimation rule. The announcement set of agent $i$ is

$$
\mathscr{F} \times(\mathscr{N} \times \mathscr{N})
$$

so agent announces a finite set of cost structures and, a connnection demand for herself. Let $A_{i}$ be announcement of agent $i$. The cost allocation rule is a map $\phi: \mathscr{U} \rightarrow \mathbb{R}_{+}^{m}$. The estimation rule is a map $C^{e}: \prod_{i} \operatorname{pr}_{\mathscr{K}} A_{i} \rightarrow \mathscr{K}$ where $C^{e}\left(\prod_{i} \operatorname{pr}_{\mathscr{K}} A_{i}\right) \in \operatorname{co}\left(\cap_{i} \operatorname{pr}_{\mathscr{K}} A_{i}\right)$ for $\cap_{i} \operatorname{pr}_{\mathscr{K}} A_{i} \neq \emptyset$ with $\operatorname{co}(\cdot)$ being the convex hull operator. Therefore if everybody is telling the truth, then the estimated cost structure is the true cost structure because $\cap_{i} \delta_{i}(P, C)=(P, C)$. Moreover the estimation rule is supposed to be sensitive in the sense that

$$
c_{j k}^{e}\left(\mathscr{K}_{i}, \prod_{i^{\prime} \neq i} \mathscr{K}_{i^{\prime}}\right)>c_{j k}^{e}\left(\mathscr{K}_{i}^{\prime}, \prod_{i^{\prime} \neq i} \mathscr{K}_{i^{\prime}}\right)
$$


for all $\mathscr{K}_{i}$ and $\mathscr{K}_{i}^{\prime}$ with $\min _{K \in \mathscr{K}_{i}} k_{j k}>\min _{K \in \mathscr{K}_{i}^{\prime}} k_{j k}$ and $\max _{K \in \mathscr{K}_{i}} k_{j k}>\max _{K \in \mathscr{K}_{i}^{\prime}} k_{j k}$.

For a list of individual announcements $A=\left(A_{i}\right)$, let $P(A)=\left(\operatorname{pr}_{\mathscr{N} \times \mathscr{N}} A_{i}\right)_{i}$ be the list of individual announcements of connection demands. If the state is $(P, C)$ and the list of individual announcements is $\left(A_{i}\right)_{i}$, then average cost of agent $i$ is

$$
\sum_{g \in \mathscr{M} \mathscr{C} \mathscr{N}\left(P(A), C^{e}\left(\prod_{i} \operatorname{pr}_{\mathscr{K}} A_{i}\right)\right)} \frac{1}{\mid \mathscr{M} \mathscr{C} \mathscr{C} \mathscr{N}\left(P(A), C^{e}\left(\prod_{i} \operatorname{pr}_{\mathscr{K}} A_{i}\right) \mid\right.} \phi_{i}(g, P(A), C)
$$

and the expected cost of agent $i$ is

$$
\sum_{s \in \delta_{i}(P, C)} \frac{\mu_{i}(s)}{\sum_{s^{\prime} \in \delta_{i}(P, C)} \mu_{i}\left(s^{\prime}\right)} \sum_{g \in \mathscr{M} \mathscr{C} \mathscr{C} N\left(P(s), C^{e}\left(\prod_{i^{\prime}} \operatorname{pr}_{\mathscr{K}} s_{i^{\prime}}\right)\right)} \phi_{i}\left(g, P(A), C^{e}\left(\prod_{i^{\prime}} \operatorname{pr}_{\mathscr{K}} s_{i^{\prime}}\right)\right) .
$$

For a cost allocation problem with incomplete information a strategy for agent $i$ is a map from the set of states to the set of announcements

$$
f_{i}: \mathscr{P} \times \mathscr{C} \rightarrow \mathscr{F} \times(\mathscr{N} \times \mathscr{N})
$$

that is measurable with respect to $\delta_{i}$ so $f_{i}(s)=f_{i}\left(s^{\prime}\right)$ provided $\delta_{i}(s)=\delta_{i}\left(s^{\prime}\right)$. A Bayesian Nash equilibrium is a list of individual strategies such that no agent can lower her expected cost.

Corollary 5 Suppose an allocation rule is simple and an estimation rule is continuous and sensitive. Then truth-telling is a Bayesian Nash equilibrium if and only if $\gamma$ is increasing in total costs and $\gamma_{i}\left(\left(a_{i}, b_{i}\right), P_{-i}, \mathscr{N}, v\right)=\gamma_{i}\left(\left(\alpha_{i}, \beta_{i}\right), P_{-i}, \mathscr{N}, v\right)$ for all $i, P_{-i},\left(a_{i}, b_{i}\right),\left(\alpha_{i}, \beta_{i}\right)$ and $v$

Proof: Assume $\gamma$ is increasing in total cost and $\gamma_{i}\left(\left(a_{i}, b_{i}\right), P_{-i}, \mathscr{N}, v\right)=\gamma_{i}\left(\left(\alpha_{i}, \beta_{i}\right), P_{-i}, \mathscr{N}, v\right)$ for all $i, P_{-i},\left(a_{i}, b_{i}\right),\left(\alpha_{i}, \beta_{i}\right)$ and $v$. Suppose that all agents use truth-telling as strategies. Then the planner uses the true connection structure and cost structure so the planner selects a MCCN. Therefore no agent can lower her expected cost by deviating.

Assume $\gamma$ is not increasing in total cost or $\gamma_{i}\left(\left(a_{i}, b_{i}\right), P_{-i}, \mathscr{N}, v\right) \neq \gamma_{i}\left(\left(\alpha_{i}, \beta_{i}\right), P_{-i}, \mathscr{N}, v\right)$ for some $i, P_{-i},\left(a_{i}, b_{i}\right),\left(\alpha_{i}, \beta_{i}\right)$ and $v$. Since complete information is a special case of incomplete information, it follows from the proof of Theorem 2 that there exists a connection problem with complete information $(C, P)$ for which truth-telling is not a Nash equilibrium. Moreover there exists $\varepsilon>0$ such that for connection problems with incomplete information, if $\max \left\{\left|C_{1}-C\right|, \ldots,\left|C_{\ell}-C\right|\right\}<\varepsilon$ and $\mu_{i}\left(P^{\prime}, C^{\prime}\right)<\varepsilon$ for all $i$ and $\left(P^{\prime}, C^{\prime}\right)$ with $P^{\prime} \neq P$, then some agent can benefit by making false announcements.

In Hougaard and Tvede (2012) we provide an example of the MCST model with incomplete information where there is a Bayesian Nash equilibrium resulting in a network that is not a MCCN. In the MCST model connection demands are known. Therefore the example shows that (1) in Theorem 3 does not generalize from complete information to incomplete information. 


\section{The German Hansa}

To emphasize that the $\mathrm{CN}$ model has broad relevance we use the German Hansa as an illustrative example of network building and cost allocation in practice. A thorough study of the Hansa is found in Dollinger (1970). The Hansa started in the middle of the 12th century as an association of north German merchants, developed into a community of cities in the middle of the 14th century and dissolved in the middle of the 17th century. The Hansa was used for obtaining trading privileges for its mechants as well as protecting and supporting its merchants. Most of the trade involving merchants with Hanseatic trading privileges took place in the area between Novgorod in east and London in west and Cologne in south and Bergen in north. The Hansa is an example of a network with locations being towns whose merchants had Hanseatic privileges and their marketplaces and connections being roads between towns and markets.

In the middle of the 12th century north German merchants regularly visiting or permanently settling in Gotland formed a community. These merchants wanted to take advantage of commercial oppotunities in Russia in Novgorod as well as in Polotsk, Vitebsk and Smolensk on the river Dvina. However pagan habitants of Finland and the Baltic countries made trade very risky. Around 1200 bishop Albert led a crusade into the Baltic countries. The Gotland community contributed to the crusade by equipping hundreds of crusaders and providing transportation. Lübeck supported the crusade. In 1241 Hamburg and Lübeck agreed to share the cost of keeping the roads between the two towns free from brigands. Both the crusade and the fight against brigandage can be seen as establishing new connections and establishing these connections can be seen as a public good for merchants with Hanseatic privileges as well as (almost) everybody else.

The German Hansa lived with the tension between Individual Rationality and truthful implementation. In 1284 the Norwegian king restricted the Hanseatic privileges. The Hansa responded with a blockade. Bremen did not participate in the blockade because being part of the blockade would have favoured Baltic towns trading with Norway. The Hansa punished Bremen by exclusion. The behaviour of Bremen can be interpreted as an attempt to free ride: if the blockade failed, Bremen could continue to trade with Norway; and, if the blockade succeeded, Bremen could benefit from the improved Hanseatic privileges. The Hansa and Denmark were at war from 1367 to 1369 when Denmark asked for peace. The Westphalian towns including Cologne traded mainly with England and the low countries. These towns did not contribute or support the war, but the Hansa did not exclude them. The lack of support from the Westphalian towns simply reflected their lack of commercial interests in the war.

The German Hansa could sanction participants by use of fines, confiscation and exclu- 
sion, but as mentioned earlier participation was voluntarily. Based on the difference between how Bremen and the Westphalian towns were treated it appears the Hansa dealt with the incompatibility of voluntary participation and truthful implementation by accepting that cost shares should depend on commercial interests or connection demands in the $\mathrm{CN}$ model. Since merchants from different towns met at marketplaces they probably knew the commercial interests of each other. Therefore (1) in Theorem 3 rather than Theorem 2 describes the relevant situation with the planner being the German Hansa. Hence the German Hansa could let cost shares depend on commercial interests such that towns with no commercial interest would not have to contribute and towns with commercial interests would have to contribute or face sanctions.

\section{Final remarks}

Extensions: Using the idea of routing-proofness from Moulin (2014) every agent $i$ could misrepresent her connection demand by splitting into $m_{i}$ aliases who each reports a connection demand. The characterization in Theorem 2 of allocation rules that truthfully implements MCCNs remains valid provided the condition in Theorem 2 is modified to

$$
\gamma_{i}\left(\left(a_{i}, b_{i}\right), P_{-i}, \mathscr{N}, v\right) \leq \sum_{k=1}^{m_{i}} \gamma_{i k}\left(\left(\alpha_{i \ell}, \beta_{i \ell}\right)_{\ell=1}^{m_{i}}, P_{-i}, \mathscr{N}, v\right)
$$

The connection demand of every agent $i$ could consist of $m_{i}$ pairs of locations $\left(a_{i}^{k}, b_{i}^{k}\right)_{k=1}^{m_{i}}$ rather than a single pair $\left(a_{i}, b_{i}\right)$. The characterizations in Theorem 1 and Corollaries 2-4 of allocation rules as well as the characterizations in Theorem 2 and Corollary 4 of allocation rules that truthfully implements MCCNs remain valid. Indeed our proofs of Theorems 1 and 2 and Corollaries 1-4 remain valid without any changes.

Summing up: For the CN model with undemanded locations: we have characterized allocation rules satisfying UII or SUII and NI as well as UII or SUII, NI and SI; we have shown that if an allocation rule implements MCCNs truthfully, then it satisfies UII and NI; and, we have characterized allocation rules satisfying UII and NI and truthfully implementing MCCNs. To our surprise it turned out that an allocation rule satisfies UII and NI if and only if relative cost shares depend on connection demands and nothing else. Consequently voluntarily participation is at odds with Individual Rationality in the $\mathrm{CN}$ model in contrast to the MCST model. Therefore economic efficiency can be attained if and only if the planner is able to prevent agents from building their own networks. 


\section{References}

Anshelevich, E., A. Dasgupta, J. Kleinberg, E. Tardos, T. Wexler \& T. Roughgarden (2008), The price of stability for network design with fair cost allocation, SIAM Journal of Computing 38, 1602-1623.

Bergantinos, G., \& L. Lorenzo (2004), A non-cooperative approach to the cost spanning tree problem, Mathematical Methods of Operations Research 59, 393-403.

Bergantinos, G., \& L. Lorenzo (2005), Optimal equlibria in the non-cooperative game associated with cost spanning tree problems, Annals of Operations Research 137, 101-115.

Bergantinos, G., \& J.J. Vidal-Puga (2010), Realizing fair outcomes in minimum cost spanning tree problems through non-cooperative mechanisms, European Journal of Operational Research 201, 811-820.

Bogomolnaia, A., \& H. Moulin (2010), Sharing a minimal cost spanning tree: Beyond the Folk solution, Games and Economic Behavior 69, 238-248.

Chen, H.-L., T. Roughgarden \& G. Valiant (2010), Designing network protocols for good equilibria, SIAM Journal of Computing 39, 1799-1832.

Dollinger, P., (1970), The German Hansa, Macmillan.

Hougaard, J.L. \& M. Tvede (2012), Truth-telling and Nash equilibria in minimum cost spanning tree models, European Journal of Operational Research 222, 566-570.

Jackson, M., (1991), Bayesian implementation. Econometrica 59, 461-477.

Juarez, R. \& R. Kumar (2013), Implementing efficient graphs in connection networks, Economic Theory 54, 359-403.

Megiddo, N., (1978), Computational complexity of the game theory approach to cost allocation for a tree, Mathematics of Operations Research 3, 189-196.

Moulin, H., (2014), Pricing traffic in a spanning network, Games and Economic Behavior 86, 475-490.

Tamir, A., (1991), On the core of network synthesis games, Mathematical Programming 50, 123-135.

Tijs, S., R. Branzei, S. Moretti \& H. Norde (2006), Obligation rules for minimum cost spanning tree situations and their monotonicity properties, European Journal of Operational Research 175, 121-134. 